\title{
Global scale transcriptome analysis of Arabidopsis embryogenesis in vitro
}

\author{
Anushka M Wickramasuriya and Jim M Dunwell
}

\begin{abstract}
Background: Somatic embryogenesis (SE) in plants is a process by which embryos are generated directly from somatic cells, rather than from the fused products of male and female gametes. Despite the detailed expression analysis of several somatic-to-embryonic marker genes, a comprehensive understanding of SE at a molecular level is still lacking. The present study was designed to generate high resolution transcriptome datasets for early SE providing the way for future research to understand the underlying molecular mechanisms that regulate this process. We sequenced Arabidopsis thaliana somatic embryos collected from three distinct developmental time-points $(5,10$ and $15 \mathrm{~d}$ after in vitro culture) using the Illumina HiSeq 2000 platform.

Results: This study yielded a total of 426,001,826 sequence reads mapped to 26,520 genes in the $A$. thaliana reference genome. Analysis of embryonic cultures after 5 and $10 \mathrm{~d}$ showed differential expression of 1,195 genes; these included 778 genes that were more highly expressed after $5 \mathrm{~d}$ as compared to $10 \mathrm{~d}$. Moreover, 1,718 genes were differentially expressed in embryonic cultures between 10 and $15 \mathrm{~d}$. Our data also showed at least eight different expression patterns during early $\mathrm{SE}$; the majority of genes are transcriptionally more active in embryos after $5 \mathrm{~d}$. Comparison of transcriptomes derived from somatic embryos and leaf tissues revealed that at least 4,951 genes are transcriptionally more active in embryos than in the leaf; increased expression of genes involved in DNA cytosine methylation and histone deacetylation were noted in embryogenic tissues. In silico expression analysis based on microarray data found that approximately $5 \%$ of these genes are transcriptionally more active in somatic embryos than in actively dividing callus and non-dividing leaf tissues. Moreover, this identified 49 genes expressed at a higher level in somatic embryos than in other tissues. This included several genes with unknown function, as well as others related to oxidative and osmotic stress, and auxin signalling.
\end{abstract}

Conclusions: The transcriptome information provided here will form the foundation for future research on genetic and epigenetic control of plant embryogenesis at a molecular level. In follow-up studies, these data could be used to construct a regulatory network for SE; the genes more highly expressed in somatic embryos than in vegetative tissues can be considered as potential candidates to validate these networks.

Keywords: Somatic embryogenesis, Transcriptomics, RNA-Seq, Arabidopsis thaliana, DNA replication, Cell cycle, Epigenetics

\section{Background}

Somatic embryogenesis (SE), where a single or a group of somatic cells differentiate to form embryonic cells under suitable in vitro conditions [1] is a good system to explore gene expression patterns associated with initial stages of embryo development. The formation of embryos from somatic cells closely resembles the developmental pathway of zygotic embryos (ZEs) and hence, the

\footnotetext{
* Correspondence: j.m.dunwell@reading.ac.uk

School of Agriculture, Policy and Development, University of Reading, Reading, UK
}

\section{Biomed Central}

molecular information generated for the SE pathway could be used to explain the dynamic molecular interactions that take place during early embryogenesis $[1,2]$. Recently, a broad analysis of SE regulation in higher plants with an especial emphasis on associated developmental pathways, differential gene expression, and proteomics has been reviewed elsewhere [3,4].

Over the past few years, progress of molecular techniques have immensely contributed in understanding the molecular aspects of SE in many plant species i.e. Arabidopsis [5,6], cotton [7], alfalfa [8], conifer [9], potato [10], Glycine 
$\max [11]$, oil palm [12,13], maize [14], Picea glauca [15], P. balfouriana [16], Vitis vinifera [17], Medicago truncatula [18] and Manihot esculenta [19,20]. Although the draft molecular interaction network provided for SE highlights the potential interactions at a molecular level [21], the functional characterization of the majority of genes involved in somatic-to-embryogenic transition and subsequent embryo maturation still remain largely unknown.

The advent of high throughput genomic and transcriptomic approaches has created great interest in the development of networks for plant metabolic processes to study how genes or gene products are regulated spatially and temporally to achieve cellular demands. Studies based on large scale transcriptome profiling have given a fundamental insight into the aspects of co-expressing genes and their roles in metabolic pathways. For instance, gene expression during the course of Arabidopsis ZE development, from zygote to mature embryos have been studied using microarray technology by Xiang et al. [22]. Recently, a global scale transcriptomic profiling of developing embryos using RNA sequencing (RNA-Seq) has been reported for the monocot model plant, Oryza sativa [23]. Moreover, Illumina RNA-Seq platform has been successfully used in a study related to developing seeds of maize [24].

Although, large scale genetic resources have been generated for Arabidopsis ZE development, a high resolution dataset for SE is still not available. Such a dataset is required to develop a system level model which will facilitate better understating of the molecular aspects of embryo development in vitro. Therefore, the present study was designed to generate transcript datasets for early stage somatic embryos through high-throughput Illumina HiSeq 2000. These data may provide a solid framework for future studies to investigate molecular interactions in early SE.

\section{Results and discussion}

Understanding the molecular mechanisms that drives plant embryogenesis is a major challenge due to limited accessibility to the developing embryos i.e. early stages of Arabidopsis ZEs. Given the advantage of highthroughput sequencing systems as well as the advanced knowledge of the ZE development pathway, availability of a standard protocol to induce somatic embryos and accessibility to the well annotated genome information, directed us to select the model plant, Arabidopsis, to study the global gene expression patterns in plant SE through RNA-Seq. The transcriptome information given here would serve as a useful resource in future to develop a regulatory system for this dicot model species; this will pave the way for genetic improvement of SE in other dicot species.

\section{Illumina sequencing and mapping sequence reads to the reference genome}

Transcriptome sequencing through RNA-Seq is a relatively straight forward approach that allows exploring gene expression profiles at a global scale. To provide a comprehensive overview of Arabidopsis SE at a transcriptional level, we sequenced cDNA libraries constructed from three distinct in vitro embryo developmental time-points using the Illumina HiSeq 2000 platform. This produced a total of $605,027,558$ sequence reads, encompassing 30,251 Mbases from all four cDNA libraries, embryos after $5 \mathrm{~d}$ (SE_5D), $10 \mathrm{~d}$ (SE_10D), 15 $\mathrm{d}$ (SE_15D) of in vitro culture and wild type (WT) leaf tissues (WT_L). On average $90.8 \%$ of the quality filter passed reads generated for all three somatic embryo samples were mapped uniquely to the reference genome. Another $2.4 \%$ of reads were mapped to multiple locations in the genome and the remaining reads were either unmapped $(2.1 \%)$ or did not show primary hits $(4.7 \%)$. Of the mapped reads identified in both tissue types, on average approximately $98.5 \%$ of reads were mapped to the exons, $0.9 \%$ of reads were mapped to introns and the remaining $0.5 \%$ of reads were aligned with the $10 \mathrm{~kb}$ upstream and downstream of the transcripts. A summary of mapping statistics obtained for each sample is given in Table 1 .

The average coverage profiles computed for the mapped reads using the Integrative Genomic Viewer (IGV) tool for each chromosome showed that the read coverage of each chromosome is uniform across all samples examined. Log transformed (base 2) coverage values were used to generate coverage plots as it gives a better visualization of read depths. Only the chromosome 1 coverage map is shown here (Figure 1).

Conversion of mapped and assembled read counts into normalized digital transcript levels (Fragments Per Kilobase of exon per Million fragments mapped (FPKM)) is a prerequisite for comparing expression profiles of genes within or between samples to provide a comprehensive overview of transcriptomes. For downstream gene expression analysis, expression measures of individual gene isoforms were combined to obtain the final transcript level for a particular gene. In this study we report the presence of transcripts for 26,520 annotated genes in at least one of the three somatic embryo samples examined; this reflects approximately $\approx 80 \%$ of the annotated genes reported in the latest Arabidopsis genome release (Additional file 1). Among these genes, 24,097 of protein coding genes (91\%), 1,338 (5\%) transposable elements (TEs), $466(2 \%)$ pseudo genes and $619(2 \%)$ other RNA genes were identified. The distribution of FPKM values for each sample analysed showed a similar pattern, skewing to the right (Figure 2).

In brief, 24,081 (73\%), 25,347 (77\%), 24,944 (75\%) and $23,675(72 \%)$ annotated genes were transcriptionally 
Table 1 Mapping statistics for quality filtered reads generated for embryogenic and leaf tissues

\begin{tabular}{lllll}
\hline & SE_5D & SE_10D & SE_15D & WT_L \\
\hline Total number of QC-passed reads & 73882504 & 212208700 & 139910622 & 179025732 \\
Total Number of mapped reads & 75839181 & 222139250 & 148769022 & 183167937 \\
Mapped percentage (\%) & 97.82 & 98.18 & 97.24 & 99.10 \\
Read -1* & 37996562 & 111267307 & 74522675 & 91775211 \\
Read -2* & 37842619 & 110871943 & 74246347 & 91392726 \\
Total number of unmapped reads & 1613269 & 3862501 & 2560237 & 1613269 \\
Un-mapped percentage (\%) & 2.18 & 1.82 & 2.76 & 0.90 \\
\hline
\end{tabular}

*read-1 and read-2: forward and reverse primer derived reads.

active in SE_5D, SE_10D, SE_15D and WT_L, respectively. The Figure 3 summarizes the distribution of expressed genes found in each sample examined. Based on our study, it was noted that a total of 3,523 genes (2,469 protein coding genes and 723 TEs) are expressed only in embryogenic tissues but not in WT leaf tissues. This included 200 transcription factor (TF) encoding genes over 31 TF families. MYB (30 genes), MADS-box (23), C3H (19), basic helix-loop-helix (bHLH) (18), C2H2 (17), homeobox (12) and APETALA2 (AP2)/ethylene responsive element binding proteins (EREBP) (11) were the main TF families observed.

Furthermore, the Gene Ontology (GO) enrichment analysis of this gene subset identified 1,864 genes with annotated GO terms. They were significantly enriched for $132 \mathrm{GO}$ terms over three main functional categories at $\mathrm{p}<0.05$, biological processes (BP) (93), molecular function (MF) (31) and cellular components (CC) (8). The highly enriched GO terms $\left(\mathrm{p}<10^{-9}\right)$ found in each main functional category is shown in Figure 4. For instance, embryo sac development (GO:0009553), megagametogenesis (GO:0009561), gametophyte development (GO:0048229), developmental process (GO:0032502), tetrapyrrole binding (GO:0046906), heme binding (GO:0020037), iron ion binding (GO:0005506), endomembrane system (GO:0012505), cell part (GO:0044464) and cell (GO:0005623) were the main functional subgroups that showed highest significance. To determine the biological pathways that are functionally more active, the specifically transcribed gene subset $(3,523)$ found in somatic embryos was further analysed through the SkyPainter tool (Arabidopsis Reactome). This showed over-representation of 17 main biological pathways (Table 2).

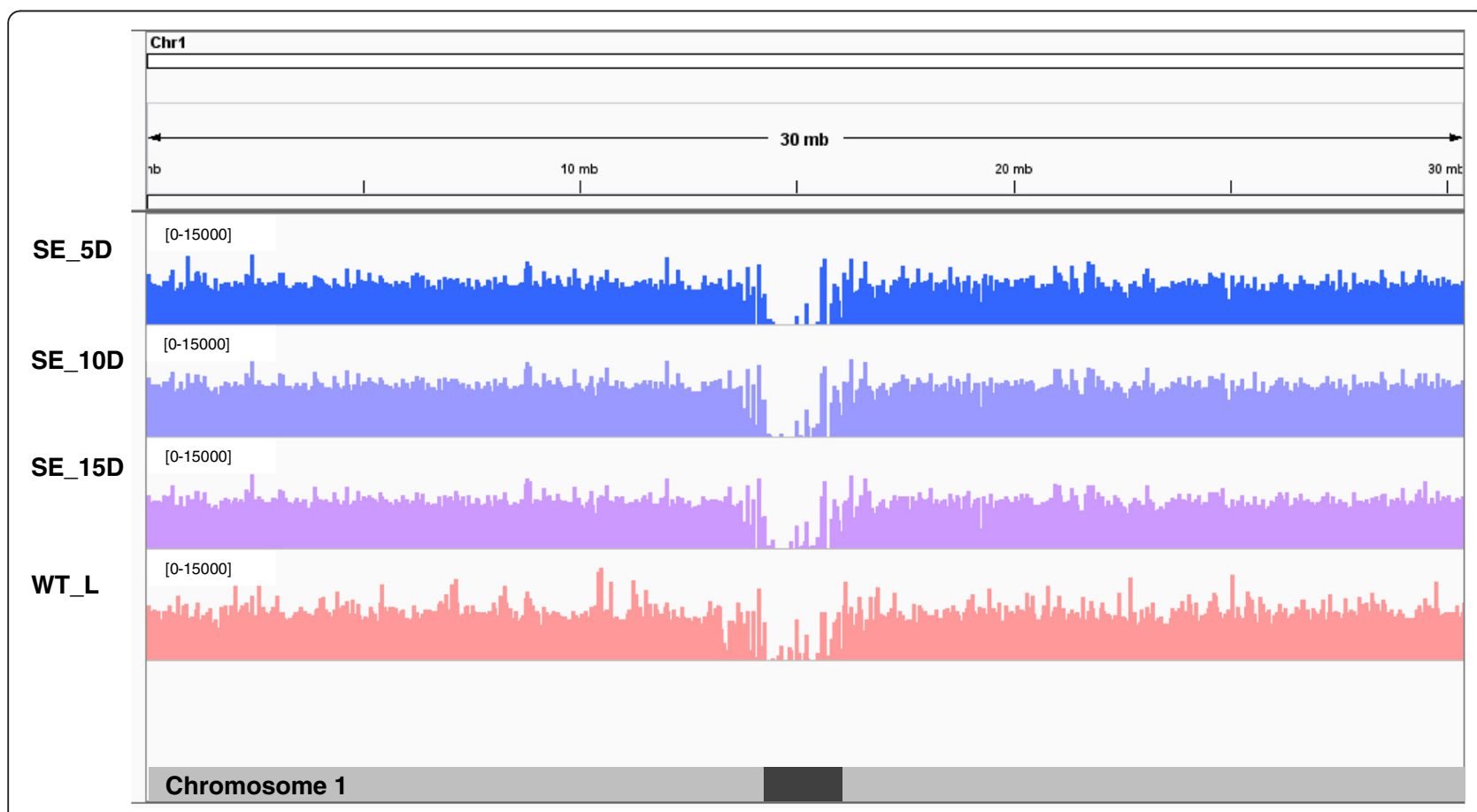

Figure 1 Coverage plots generated for transcripts aligned to the chromosome 1. SE_5D, SE_10D and SE_15D are to represent embryogenic samples collected after 5, 10 and $15 \mathrm{~d}$ of in vitro culture, respectively. WT_L: WT leaf tissues. 


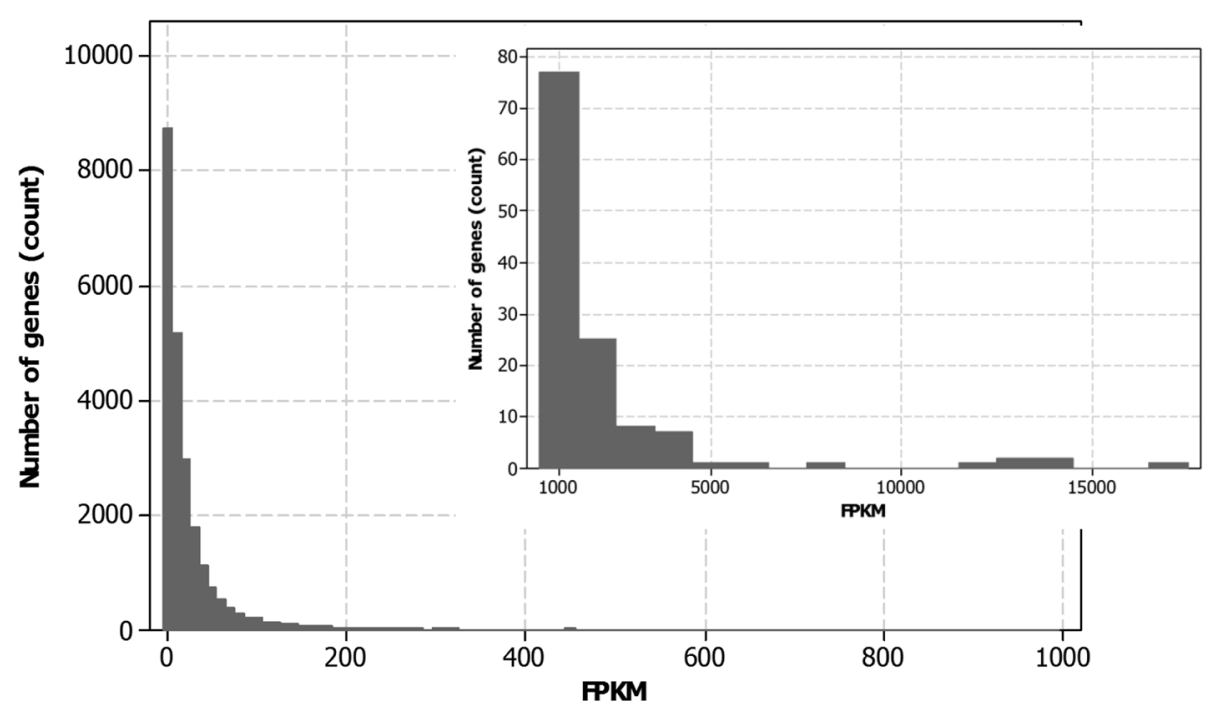

Figure 2 Distribution of transcript abundance in somatic embryo transcriptomes. This represents the transcriptome of embryogenic tissues collected after $5 \mathrm{~d}$ of in vitro culture.

In general, as compared to previous plant embryogenesis related studies, our study report abundant transcript information for downstream analysis. For example, an expression analysis based on microarray technique has demonstrated expression of at least 22,800 genes across the three embryonic stages, globular, heart and torpedo in Arabidopsis [25]. A similar expression analysis has shown expression of 17,594 different transcripts in at least one of the stages or sub-regions of Arabidopsis developing seeds [26]. Therefore, the Arabidopsis somatic embryo transcriptome information given here may serve as a valuable resource for future studies.

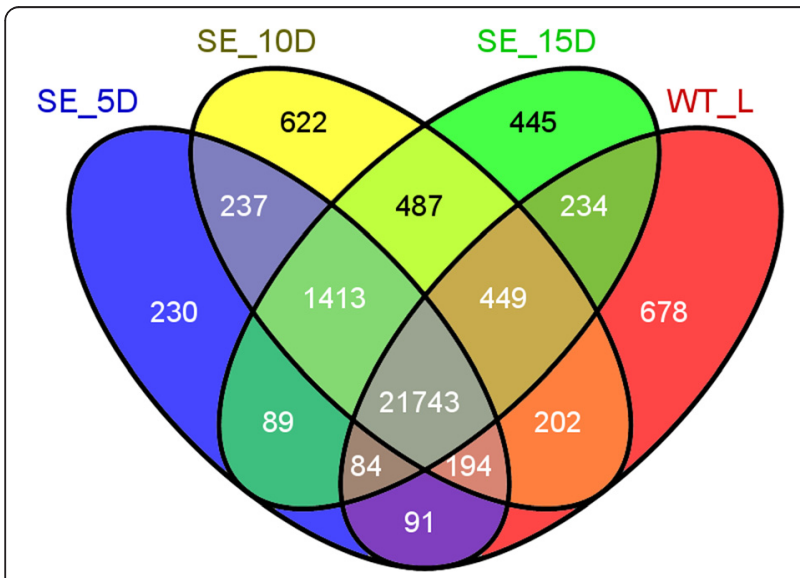

Figure 3 Venn diagram representing the transcribed genes detected in embryogenic and leaf tissues. SE_5D, SE_10D and SE_15D are to represent embryogenic samples collected after 5, 10 and $15 \mathrm{~d}$ of in vitro culture, respectively. WT_L: WT leaf tissues.
Gene expression patterns across the three distinct in vitro embryo developmental time-points

To provide an overview of potential gene expression patterns that may exist during in vitro embryogenesis, transcript levels of 26,520 annotated genes were compared between different embryo developmental timepoints. Of these, 23,156 genes were co-expressed in all three developmental time-points; 431 were co-expressed between SE_5D and SE_10D; 936 were co-expressed between SE_10D and SE_15D; 173 were co-expressed between SE_5D and SE_15D. In addition, 321, 824 and 679 genes were preferentially expressed in SE_5D, SE_10D and SE_15D, respectively. Although a considerable fraction of co-expressed genes was identified between time-points, the most of the genes showed noticeable variations in transcript levels. Thus, to determine differentially expressed genes (DEGs), transcript levels (FPKM) of genes were compared between the timepoints. A total of 1,195 DEGs were found between 5 and $10 \mathrm{~d}$ after in vitro culture with 417 up-regulated (Log2 [fold change $(\mathrm{FC})] \geq 2.0)$ and 778 down-regulated (Log2 $[\mathrm{FC}] \leq-2.0)$ genes. It was found that $82(7 \%)$ TF encoding genes are differentially expressed between these two timepoints and the majority were from TF families such as MYB (14), bHLH (12) and MADS (9) (Table 3). Additionally, several embryogenesis related genes such as LATE EMBRYOGENESIS ABUNDANT (LEA) genes (AT1G54890, AT1G64065, AT3G19430, AT2G35970, AT4G27400, AT1G61760, AT2G40170, AT5G60530 and AT5G54370), nitrate transport 1.6 (AT1G27080), AGAMOUS-like 81 (AGL81; AT5G39750), MATERNAL EFFECT EMBRYO ARREST 27 (AT2G34880), MATERNAL EFFECT EMBRYO ARREST 8 (AT1G25310), ATS3-like 


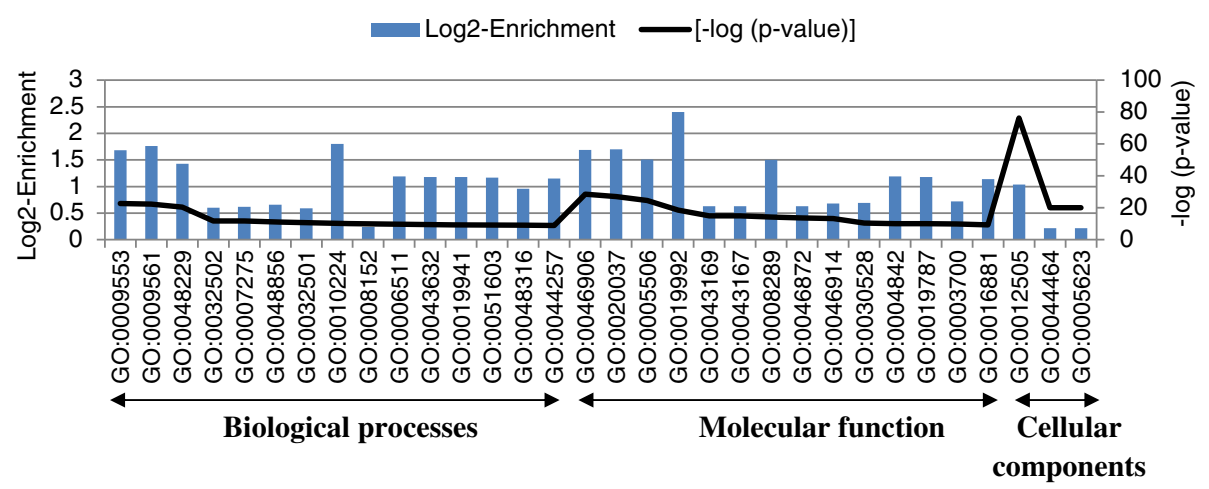

Figure 4 More highly enriched GO terms found within the gene subset that showed a somatic embryo specific expression pattern. GO:0009553: embryo sac development; GO:0009561:megagametogenesis; GO:0048229: gametophyte development; GO:0032502: developmental process; GO:0007275: multicellular organismal development; GO:0048856: anatomical structure development; GO:0032501: multicellular organismal process; GO:0010224: response to UV-B; GO:0008152: metabolic process; GO:000651 1: ubiquitin-dependent protein catabolic process; GO:0043632: modification-dependent macromolecule catabolic process; GO:0019941: modification-dependent protein catabolic process; GO:0051603: proteolysis involved in cellular protein catabolic process; GO:0048316: seed development; GO:0044257: cellular protein catabolic process; GO:0046906: tetrapyrrole binding; GO:0020037: heme binding; GO:0005506: iron ion binding; GO:0019992: diacylglycerol binding; GO:0043169: cation binding; GO:0043167: ion binding; GO:0008289: lipid binding; GO:0046872: metal ion binding; GO:0046914: transition metal ion binding; GO:0030528: transcription regulator activity; GO:0004842: ubiquitin-protein ligase activity; GO:0019787: small conjugating protein ligase activity; GO:0003700: transcription factor activity; GO:0016881: acid-amino acid ligase activity; GO:0012505: endomembrane system; GO:0044464: cell part; GO:0005623: cell.

gene (AT5G62210), EMBRYO SAC DEVELOPMENT ARREST 36 (AT4G13890), ATS1 (AT4G26740) and EMBRYO SAC DEVELOPMENT ARREST 24 (AT1G70540) were also detected among the DEGs.

A similar differential gene expression analysis between 10 and $15 \mathrm{~d}$ after in vitro cultures identified a total of

Table 2 Over-represented biological pathways found within the specifically transcribed gene subset detected in embryogenic tissues

\begin{tabular}{ll}
\hline Plant biological pathway & Probability \\
\hline Glucosinolate biosynthesis from tryptophan & $2.50 \mathrm{E}-03$ \\
Glucosinolate biosynthesis from phenylalanine & $2.70 \mathrm{E}-03$ \\
Glucosinolate biosynthesis from homomethionine & $2.70 \mathrm{E}-03$ \\
Anthocyanin biosynthesis & $2.90 \mathrm{E}-03$ \\
Gibberellin biosynthesis III (early C-13 hydroxylation) & $3.30 \mathrm{E}-03$ \\
Cytokinins-O-glucoside biosynthesis & $4.10 \mathrm{E}-03$ \\
Cytokinins 7-N-glucoside biosynthesis & $4.10 \mathrm{E}-03$ \\
Cytokinins 9-N-glucoside biosynthesis & $4.10 \mathrm{E}-03$ \\
ent-kaurene biosynthesis & $4.30 \mathrm{E}-03$ \\
(deoxy)ribose phosphate degradation & $1.40 \mathrm{E}-02$ \\
Abscisic acid biosynthesis & $1.80 \mathrm{E}-02$ \\
de novo biosynthesis of purine nucleotides II & $1.90 \mathrm{E}-02$ \\
Gibberellin biosynthesis I (non C-3, non C-13 & $2.10 \mathrm{E}-02$ \\
hydroxylation) & \\
Gibberellin biosynthesis II (early C-3 hydroxylation) & $2.10 \mathrm{E}-02$ \\
Gibberellin inactivation & $2.10 \mathrm{E}-02$ \\
de novo biosynthesis of purine nucleotides I & $7.60 \mathrm{E}-02$ \\
Trans-zeatin biosynthesis & $9.50 \mathrm{E}-02$ \\
\hline
\end{tabular}

1,067 up regulated genes and 651 down regulated genes. This comprised 114 (7\%) TF genes; the majority were members of TF families such as MYB or MYB related (20 genes), MADS-box (12) and AP2/EREBP (12) (Table 3). Furthermore, LEA genes such as AT1G61760, AT5G22870, AT5G53730, AT3G02480, AT1G52680, AT5G54370, AT4G36600, AT3G22500, AT5G06760, AT3G15670, AT2G35300, AT4G27400, AT1G32560 and $A T 1 G 72100$ as well as several other embryogenesis related genes i.e. EMBRYO SAC DEVELOPMENT 39, SEED GENE 3 (ATS3), AGL81, RESPONSIVE TO ABSCISIC ACID 28 (RAB28; AT1G03120) were also differentially expressed between SE_10D and SE_15D.

Visualization of functional networks generated based on enriched GO terms related to BP for the identified DEGs between different somatic embryo developmental timepoints exhibited the complexity of responses between the time-points. For example, genes expressed at a noticeably higher level in embryogenic tissues after $5 \mathrm{~d}$ as compared $10 \mathrm{~d}$ were mainly enriched for functional networks related to hormone transport (i.e. regulation of polar auxin transport), carbohydrate mediated signalling pathways and cell wall organization and biogenesis (Figure 5). In contrast, more highly expressed genes found in embryogenic cultures after $10 \mathrm{~d}$ were mainly enriched for functional networks related to energy metabolism and regulation of various responses to stimuli. A similar analysis of genes up regulated between embryogenic tissues after 10 and $15 \mathrm{~d}$ showed over-representation of biological networks such as cellular hormone biosynthesis and metabolic process (i.e. auxin, abscisic acid, and ethylene), regulation of secondary metabolic process and regulation of reproductive process. 
Table 3 Top most differentially expressed TF encoding genes detected between embryo developmental time-points

\begin{tabular}{|c|c|c|c|}
\hline Gene ID & Log2 [FC] & TF family & Gene description \\
\hline \multicolumn{4}{|c|}{ Differentially expressed TFs between $S E_{-} 5 D$ and $S E_{-} 10 D$} \\
\hline AT5G27090 & 4.82 & MADS & Protein agamous-like 54 \\
\hline AT3G46070 & 3.58 & $\mathrm{C} 2 \mathrm{H} 2$ & $\mathrm{C} 2 \mathrm{H} 2$-type zinc finger family protein \\
\hline AT5G52600 & 3.52 & MYB & MYB82 \\
\hline AT5G40220 & 3.28 & MADS & Protein agamous-like 43 \\
\hline AT1G66420 & 3.27 & GeBP & $\begin{array}{l}\text { DNA-binding storekeeper protein-related transcriptional } \\
\text { regulator }\end{array}$ \\
\hline AT1G13260 & 3.26 & RAV & AP2/ERF and B3 domain-containing TF \\
\hline AT2G47190 & 3.09 & MYB & R2R3 MYB DNA binding domain TF (MYB2) \\
\hline AT3G56970 & 3.01 & bHLH & ORG2 (BHLH038) \\
\hline AT5G41570 & 3.00 & WRKY & WRKY transcription factor 24 \\
\hline AT5G15150 & 2.83 & Homeobox & Homeobox-leucine zipper protein HAT7 (HB-3) \\
\hline AT3G11110 & -5.91 & $\mathrm{C} 3 \mathrm{H}$ & RING-H2 finger protein ATL66 \\
\hline AT4G09960 & -4.50 & MADS & Agamous-like MADS-box protein AGL11 \\
\hline AT1G75250 & -4.06 & MYB-related & Protein RADIALIS-like 6 \\
\hline AT1G25310 & -3.79 & bHLH & Transcription factor MEE8 \\
\hline AT2G27940 & -3.73 & $\mathrm{C} 3 \mathrm{H}$ & RING-H2 finger protein ATL57 \\
\hline AT1G19510 & -3.65 & MYB-related & Protein RADIALIS-like 5 \\
\hline AT1G56650 & -3.64 & MYB & Transcription factor MYB75 \\
\hline AT3G06120 & -3.23 & bHLH & Transcription factor MUTE \\
\hline AT5G46830 & -3.22 & bHLH & Calcium-binding transcription factor NIG1 \\
\hline AT5G53200 & -3.21 & MYB-related & Transcription factor TRY \\
\hline \multicolumn{4}{|c|}{ Differentially expressed $T F s$ between $S E_{-} 10 D$ and $S E_{-} 15 D$} \\
\hline AT2G21900 & 6.41 & WRKY & WRKY DNA-binding protein 59 \\
\hline AT5G01900 & 5.04 & WRKY & WRKY DNA-binding protein 62 \\
\hline AT2G40750 & 4.80 & WRKY & WRKY DNA-binding protein 54 \\
\hline AT5G65790 & 4.78 & MYB & MYB domain protein 68 \\
\hline AT1G74080 & 4.28 & MYB & MYB domain protein 122 \\
\hline AT1G02230 & 4.28 & NAC & NAC domain containing protein 4 \\
\hline AT2G45660 & 4.27 & MADS & AGAMOUS-like 20; Involved in controlling flowering \\
\hline AT5G22570 & 4.17 & WRKY & WRKY DNA-binding protein 38 \\
\hline AT5G64810 & 4.16 & WRKY & WRKY DNA-binding protein 51 \\
\hline AT1G66600 & 4.13 & WRKY & $\begin{array}{l}\text { WRKY transcription factor } 63 \text {; Involved in regulation of } \\
\text { plant responses to ABA and drought stress }\end{array}$ \\
\hline AT2G34820 & -15.05 & $\mathrm{bHLH}$ & Basic helix-loop-helix 53 \\
\hline AT5G39750 & -7.70 & MADS & AGAMOUS-like 81 \\
\hline AT1G24260 & -4.41 & MADS & MADs box transcription factor SEPALLATA3 \\
\hline AT3G50330 & -3.80 & bHLH & HECATE 2 \\
\hline AT4G00120 & -3.67 & bHLH & INDEHISCENT \\
\hline AT5G14010 & -3.21 & $\mathrm{C} 2 \mathrm{H} 2$ & $\begin{array}{l}\text { Zinc finger protein KNUCKLES; Mediates the repression } \\
\text { of WUS in floral meristem determinacy control }\end{array}$ \\
\hline AT4G30180 & -3.00 & $\mathrm{bHLH}$ & Hypothetical protein \\
\hline AT4G26150 & -3.00 & C2C2-Gata & Putative GATA transcription factor 22 \\
\hline AT1G25250 & -2.83 & $\mathrm{C} 2 \mathrm{H}_{2}$ & Indeterminate-domain 16 \\
\hline AT4G29030 & -2.82 & Trihelix & Putative membrane lipoprotein \\
\hline
\end{tabular}




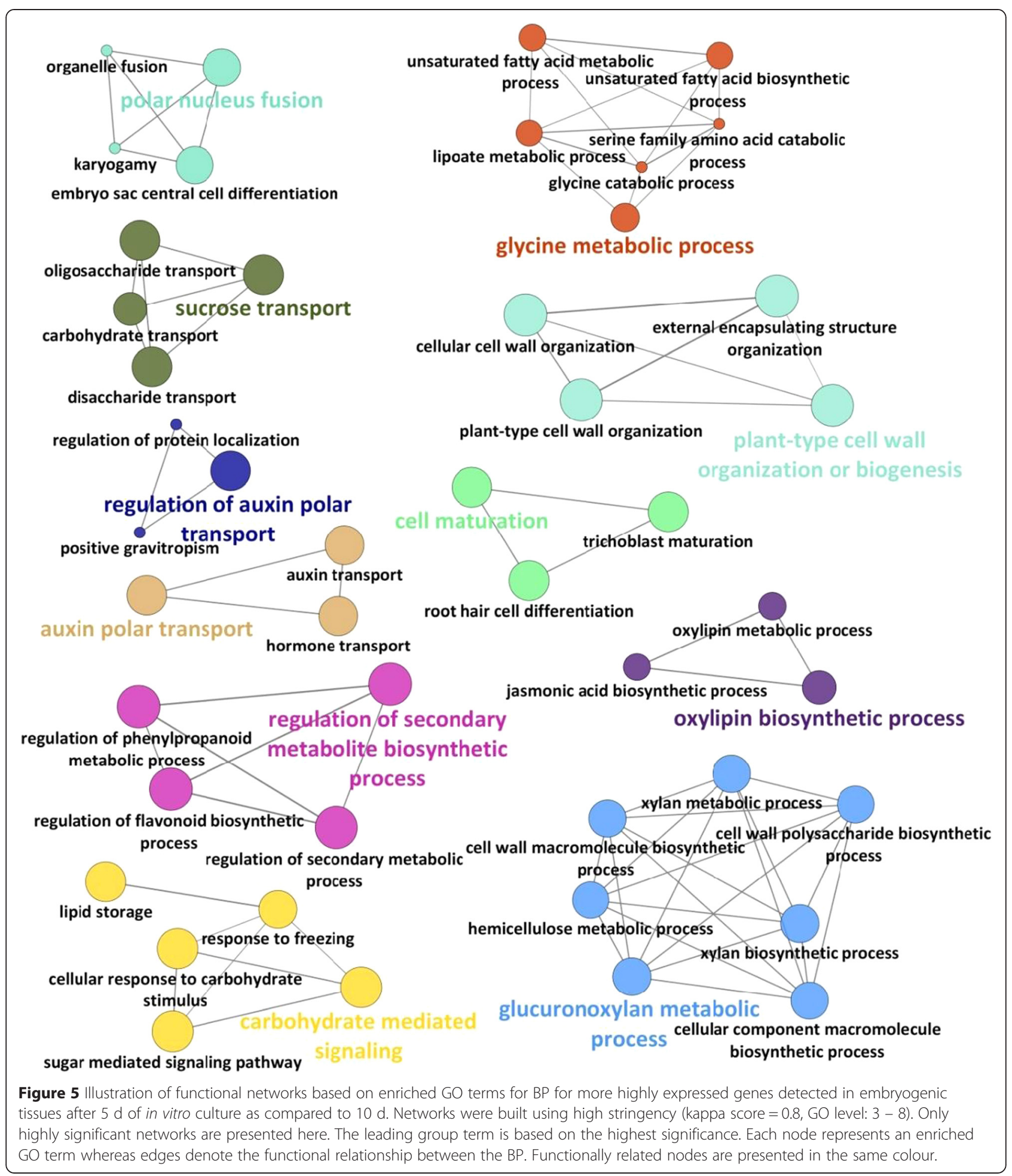

In summary, a total of 3,790 DEGs were detected in the process of in vitro embryo development; 1,195 genes between SE_5D and SE_10D; 1,718 genes between SE_10D and SE_15D; 2,817 genes between SE_5D and SE_15D. The remaining genes showed relatively stable expression during this process. In order to determine the potential gene expression patterns that may exist throughout the course of embryo development, the FPKM read counts of these DEGs were obtained for each embryo developmental time-point and transformed into a $\log$ (base 2) scale. Of these, 438 genes were excluded from the downstream cluster analysis as they 
were not expressed/detected $(\mathrm{FPKM}=0)$ in at least one of the tissue sampling time-points. The remaining 3,352 genes were grouped into 10 user defined clusters based on their expression patterns. Based on the 10 clusters generated from the software, at least eight distinct gene expression patterns were detected (Figure 6). In accordance with previous research on rice embryo development [23], the majority of genes (912) fell into the continuously
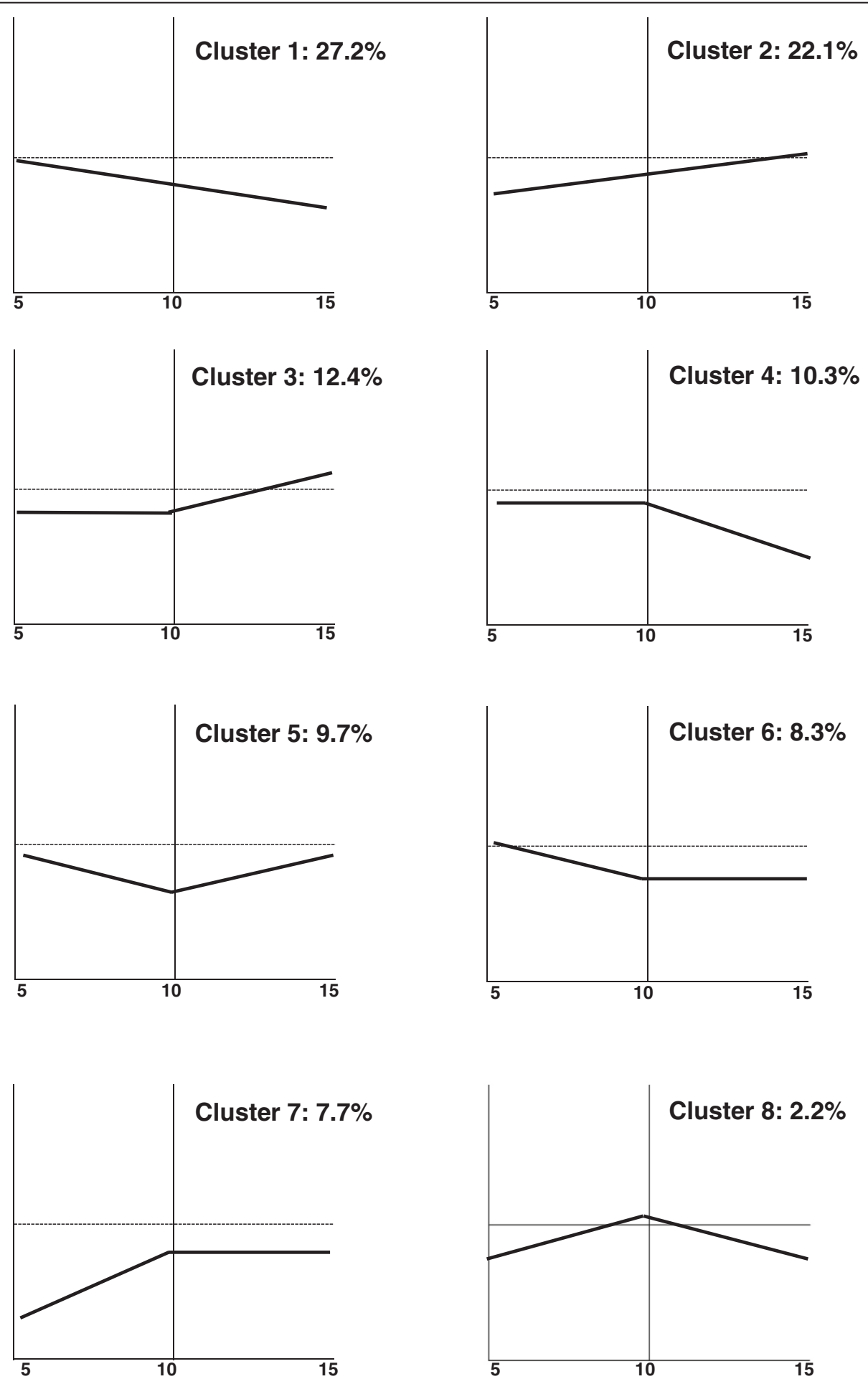

Figure 6 Potential gene expression patterns during SE. The X-axis represents somatic embryo developmental time-points, 5, 10 and $15 \mathrm{~d}$ after in vitro culture. 
down regulated gene cluster (cluster 1). These genes were highly enriched for GO terms related to carbohydrate, lipid and amino acid metabolic processes. In addition, we found that expression levels of 740 genes gradually increase with embryo maturation (cluster 2). This gene subset was mainly enriched for stress responsive genes.

The eight different expression patterns identified here were further analyzed to examine whether any of these clusters are enriched for a particular TF family. It was found that at least 265 TF genes covering $\approx 30$ different TF families are differentially expressed during SE. Figure 7A shows the distribution of TF related genes among the clusters. With the exception of clusters 2 and 4 , almost all the clusters consisted of approximately $6-7 \%$ of TF related genes. A relatively higher percentage of TF related genes was detected in cluster 2 and 4, which was about $11 \%$ and $9 \%$, respectively. Moreover, among the differentially expressed TFs detected, the majority belong to the TF families such as bHLH $(35 ; 13 \%)$, MYB (34; 13\%), AP2/EREBP (24; 9\%), MADS-box (19; 7\%), WRKY
(19: 7\%) and homeobox domain proteins (14; 5\%). The distribution of highly occurring TF families within each cluster is highlighted in Figure 7B. The distribution percentage of each TF family was calculated in relation to each TF family size given in the Arabidopsis Gene Regulatory Information Server (AGRIS, http://arabidopsis.med. ohio-state.edu/). Most of the genes related to AP2/EPEBP, homeobox, MADS-box and bHLH families were found in clusters 1 and 2 where the gene expression was relatively high at the early and late stages, respectively.

Analysis of the remaining 438 genes that showed zero FPKM in at least one of the three embryo developmental time-points mainly fell into three groups: differential gene expression only between SE_5D and SE_10 (cluster A); differential gene expression only between SE_10D and SE_15D (cluster B); differential gene expression between SE_5D and SE_15D (cluster C). In summary, a total of 131 (cluster A), 244 (cluster B) and 63 (cluster C) genes were manually grouped into each cluster. It was found that genes related to anatomical structure

(A)

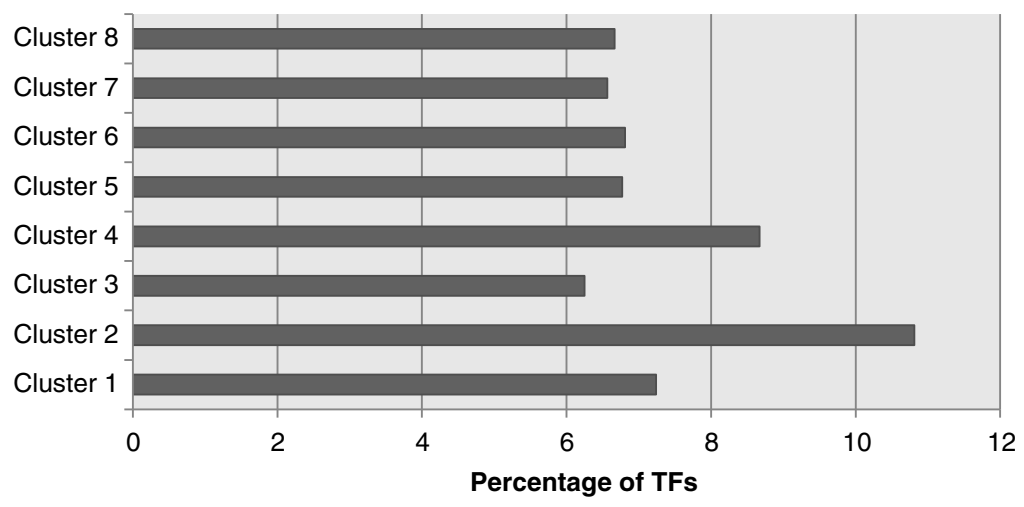

(B)

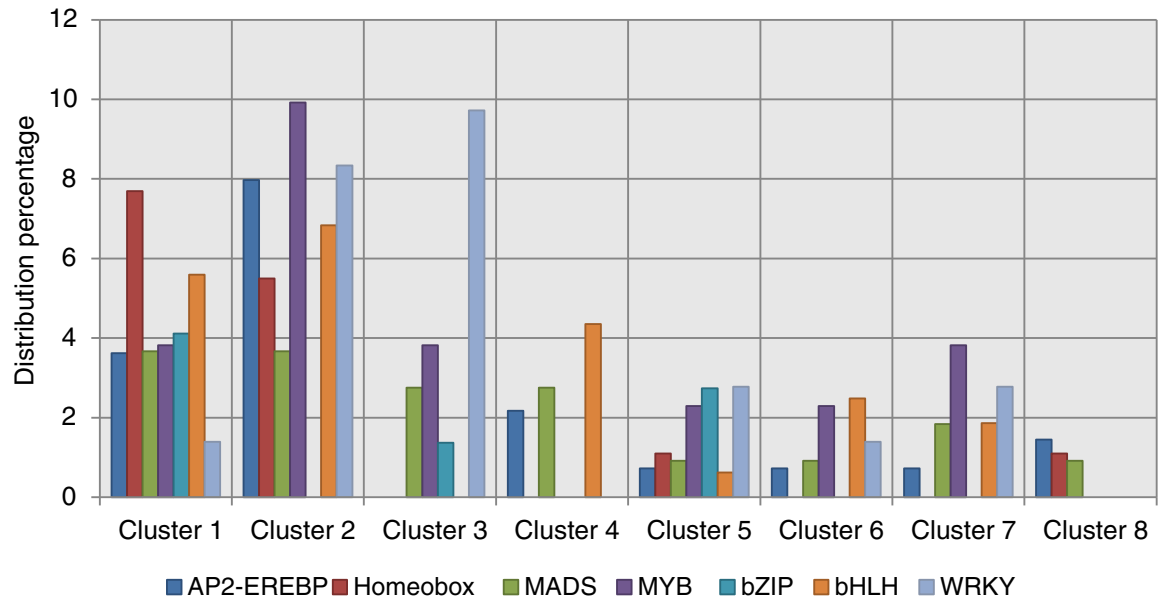

Figure 7 Distribution of TFs across the eight gene clusters. (A), Percentage of TFs in each gene cluster; (B), Distribution of TF family members across the clusters. 
development processes and catalytic activity were mainly enriched within the cluster A genes whereas genes related to peptide biosynthetic processes and nucleic acid binding were predominately enriched within cluster $\mathrm{B}$. The genes fell into cluster $C$ were largely enriched for stress responses. Additionally, these gene clusters were further analysed to examine whether any of these clusters are enriched for a particular family of TFs. Although several genes related to MADS-box, WRKY, MYBrelated and homeobox was found within the clusters, none of the clusters showed enrichment for a particular type of TF family. This may be due to the presence of limited number of member genes within each cluster.

\section{Genes identified as being more highly expressed in embryogenic tissues than in leaf tissues}

Although, a considerable fraction of co-expressed genes was detected between somatic embryos and WT leaf tissues, the majority of those genes showed a greater variation in transcript levels. Therefore, to identify more highly expressed genes in somatic embryos, transcriptomes of in vitro embryos and WT_L were compared and genes with $\log 2$ ratios $\geq 2.0$ were considered as more highly expressed genes in embryos. Based on this criterion, a total of 4,951 highly expressed genes were identified in somatic embryos; including 4,738 protein coding genes and 72 TEs. Of these, 3,896 genes were identified with annotated GO terms, enriching for 288 functional groups at $\mathrm{p}<0.05$. GO terms such as cellular process (GO: 0009987), structural constituent of ribosome (GO: 0003735) and organelle (GO: 0043228) were the dominant functional groups of BP, MF and CC, respectively. Further analysis of this gene subset to detect over-represented biological pathways identified 21 pathways with a greater significance (Table 4). For instance, genes involved in biological pathways such as cell cycle, DNA replication, endoreplication and alternative cell cycles, cell cycle checkpoints, pyrimidine metabolism and hormone biosynthesis were relatively highly expressed in somatic embryos than in WT_L.

The gene expression resources deposited in public repositories such as AtGenExpress (http://www.weigelworld. org/resources/microarray/AtGenExpress/), Genevestigator (https://www.genevestigator.com/gv/) and ArrayExpress (http://www.ebi.ac.uk/arrayexpress/) are useful to investigate expression patterns of gene(s)/gene families in variety of context i.e. expression across different tissue types, under diverse environmental conditions. We used Genevestigator expression datasets (microarray based) to further validate the expression patterns of a subset of those genes expressed more highly in somatic embryos than in leaf tissues by RNA-Seq. The resulting heat map for the embryogenic and leaf tissues revealed that the majority of genes selected are transcribed at a
Table 4 Over-represented biological pathways detected within the more highly expressed gene subset identified in somatic embryos

\begin{tabular}{ll}
\hline Biological pathway & Probability \\
\hline Cell Cycle, Mitotic & $1.10 \mathrm{E}-39$ \\
DNA Replication & $2.50 \mathrm{E}-16$ \\
Endoreduplication and Alternative cell cycles & $3.30 \mathrm{E}-13$ \\
Cell Cycle Checkpoints & $9.30 \mathrm{E}-05$ \\
Pyrimidine metabolism (KEGG) & $3.10 \mathrm{E}-04$ \\
Purine metabolism (KEGG) & $1.90 \mathrm{E}-02$ \\
Cytokinins-O-glucoside biosynthesis (AraCyc) & $2.50 \mathrm{E}-02$ \\
Cytokinins 7-N-glucoside biosynthesis (AraCyc) & $2.50 \mathrm{E}-02$ \\
Cytokinins 9-N-glucoside biosynthesis (AraCyc) & $2.50 \mathrm{E}-02$ \\
Anthocyanin biosynthesis (AraCyc) & $2.60 \mathrm{E}-02$ \\
Sucrose Biosynthesis & $2.80 \mathrm{E}-02$ \\
Methionine degradation II (AraCyc) & $2.80 \mathrm{E}-02$ \\
G1/S DNA integrity checkpoint & $2.80 \mathrm{E}-02$ \\
Glucosinolate biosynthesis from homomethionine (AraCyc) & $4.10 \mathrm{E}-02$ \\
Diterpenoid biosynthesis (KEGG) & $4.90 \mathrm{E}-02$ \\
de novo biosynthesis of purine nucleotides II (AraCyc) & $5.40 \mathrm{E}-02$ \\
Glucosinolate biosynthesis from tryptophan (AraCyc) & $6.30 \mathrm{E}-02$ \\
Glucosinolate biosynthesis from phenylalanine (AraCyc) & $6.90 \mathrm{E}-02$ \\
Sucrose degradation to ethanol and lactate (anaerobic) & $7.70 \mathrm{E}-02$ \\
(AraCyc) & \\
Sucrose biosynthesis (AraCyc) & $8.30 \mathrm{E}-02$ \\
Gibberellin biosynthesis III (early C-13 hydroxylation) & $9.10 \mathrm{E}-02$ \\
(AraCyc) & \\
\hline
\end{tabular}

higher level in embryonic samples than in leaf tissues (Additional file 2).

Additionally, to identify the genes that encode for essential and non-redundant function, the subset of genes that were expressed at a higher level in somatic embryos were probed with the embryo defective mutant gene list reported in the SeedGenes database (http://www.seedgenes. org/). This database provides information on 481 essential genes and 888 mutants. It was evident that 101 (21\%) of those genes are expressed at a higher level in our somatic embryogenic samples than in leaf samples (Additional file 3 ). These genes represent approximately $2 \%$ of the more highly expressed gene list detected in somatic embryos. Gene expression patterns based on microarray data (Genevestigator) also confirmed that the majority of these genes were expressed in reproductive tissues rather than in vegetative tissues (Additional file 2). On the other hand, our study detected 2,149 genes that were expressed at a higher level in leaf tissues (Log2 $[F C] \leq-2.0)$. Analysis of this gene subset identified 47 genes were present in the list of 481 embryo defective genes in the SeedGenes database. Further investigation 
of transcript levels using Genevestigator clearly showed that these 47 genes are expressed at a considerably higher level in leaf tissues than in embryogenic tissues (Additional file 4).

Establishment of in vitro embryogenic cultures requires activation of several specific gene subsets. Of these, genes that regulate the cell cycle and DNA replication play a key role in controlling active cell proliferation. In this study, comparative expression profile analysis of 88 core cell cycle genes $[27,28]$ in somatic embryos and WT_L revealed that at least 39 genes are transcriptionally more active during $\mathrm{SE}$ with $\log 2[\mathrm{FC}] \geq 2.0$ (Figure 8; see Additional file 5 for the expression patterns of these genes based on microarray data from Genevestigator). Of these, five cyclin dependent kinases (CDKs) (CDKB1;1, $C D K B 1 ; 2, C D K B 2 ; 1, C D K B 2 ; 2$ and $C D K D ; 1)$, seven type-A cyclins (CYCA1;1, CYCA1;2, CYCA2;1, CYCA2;2, CYCA2;3, $C Y C A 3 ; 1$ and $C Y C A 3 ; 2)$, nine type-B cyclins (CYCB1;1, CYCB1;2, CYCB1;3, CYCB1;4, CYCB2;1, $C Y C B 2 ; 2, C Y C B 2 ; 3, C Y C B 2 ; 4$ and $C Y C B 3 ; 1)$ and four
cyclin-D (CYCD3;1, CYCD3;3, CYCD4;1 and CYCD6;1) were identified. Additionally, several CDK-like (CKL) genes (CKL3, CKL8, CKL11, and CKL12) and gene members of the E2F TFs that function in cyclin D/retinoblastoma/E2F pathway i.e. E2Fa, E2Fc and E2L2 also showed noticeable accumulation of transcripts in embryogenic tissues than in WT leaf tissues. Several studies have reported involvement of key regulators of cell cycle during embryogenesis. For instance, expression analysis of a $C D K$ in coconut $\mathrm{SE}$ has shown a gradual decrease in expression of $C n C D K A$ during the course of somatic embryo development [29]. Moreover, over-expression of Arabidopsis CDKA;1 caused developmental defects in embryos and lead to embryo lethality when it is knocked out [30,31]. It has been hypothesized that cell cycle genes may closely interact with hormone and other developmental signalling pathways to regulate in vitro embryogenesis [32]. However, additional research is needed to support this conclusion. Therefore, the expression patterns presented

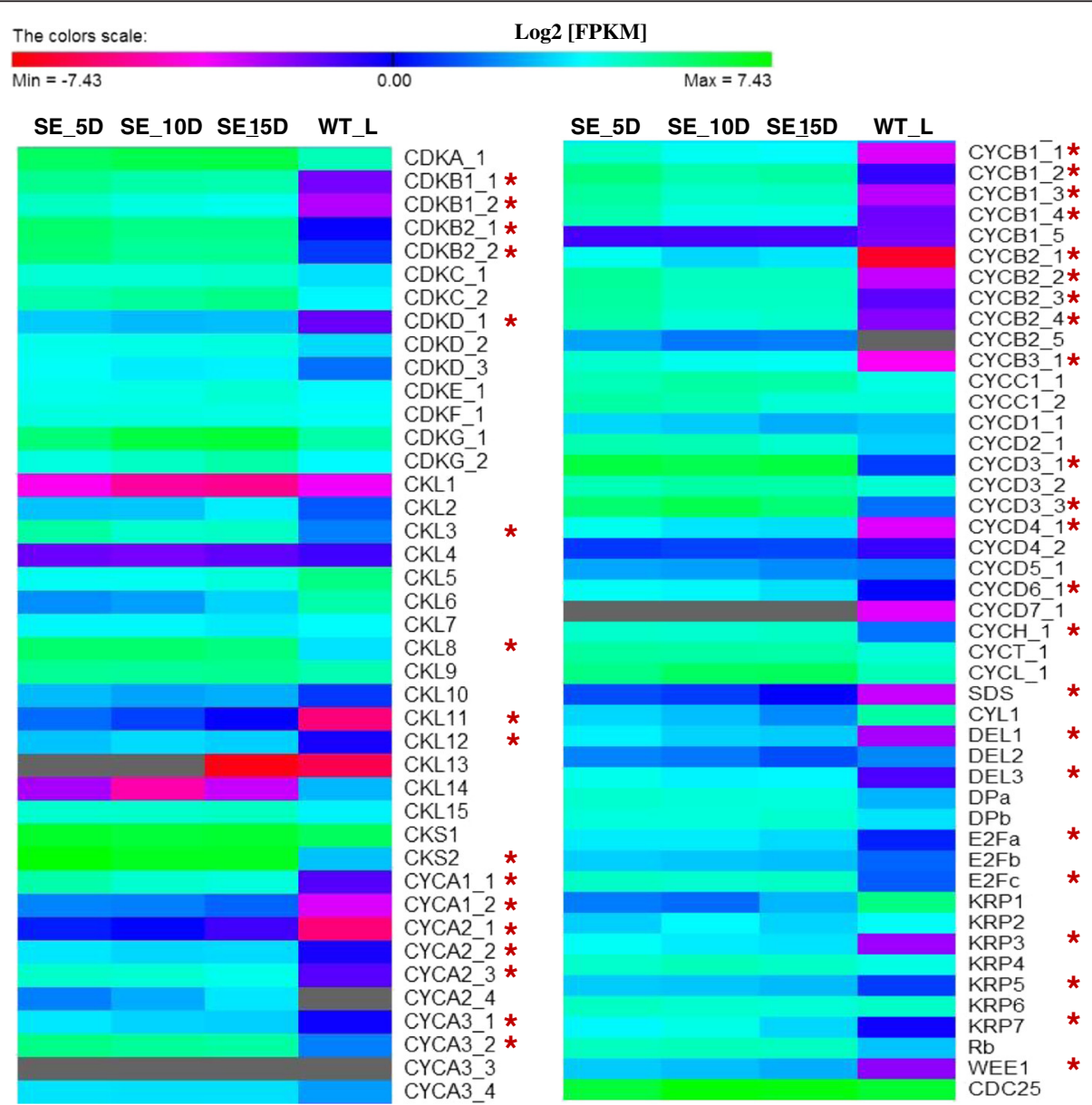

Figure 8 Transcript levels of core cell cycle related genes in embryogenic and leaf tissues. SE_5D, SE_10D and SE_15D are to represent embryogenic samples collected after 5, 10 and $15 \mathrm{~d}$ of in vitro culture, respectively. WT_L: WT leaf tissues. Ash coloured cells denote zero FPKM values (no transcripts detected). The DEGs detected between somatic embryo and leaf tissues are denoted by asterisks. 
here may be useful to inform future research to investigate the molecular function of these genes during SE.

DNA replication plays a central role in the life cycle of plants. Mutations in key genes involved in this process have caused several embryo defective phenotypes in Arabidopsis. For instance, mutations in the catalytic subunit of the DNA polymerase epsilon complex have shown developmental defects during embryogenesis [33]. Therefore, to identify transcriptionally more active DNA replication related genes in embryogenic tissues, transcript levels of 65 genes related to DNA replication machinery [34] were filtered from embryonic and leaf transcriptomes, and compared. It was found that among these genes, 48 are transcriptionally more active in somatic embryos with $\log 2[\mathrm{FC}] \geq 2.0$ (see Additional file 6 for the expression patterns of these genes based on microarray data from Genevestigator); 30 of these genes were successfully mapped to the DNA replication pathway provided by the Kyoto Encyclopedia of Genes and Genomes (KEGG) (Figure 9). This is in accordance with the finding of Masuda et al. [35], who reported higher expression of Arabidopsis pre-replication complex components (i.e. ORC1-6, CDT1a and CDC6a) in reproductive tissues than in vegetative tissues. Further analysis of transcript levels for 32 genes related to DNA double strand break (DSB) repair mechanism identified 18 significantly expressed genes in somatic embryos ( $\log 2$ $[F C] \geq 2.0$ ) (Figure 10). This included several well-studied
DSB repair genes such as GAMMA RESPONSE 1 (GR1), RAS ASSOCIATED WITH DIABETES 51 (RAD51), BREAST CANCER SUSCEPTIBLITY 1 (BRCA1), MEIOTIC RECOMBINATION 11 (MRE11) and KU70. These finding collectively suggest the importance to DNA replication and repair mechanism during SE which involves active cell proliferation.

Several TF encoding genes $(\approx 785)$ were also differentially expressed between two tissue types examined here. Of these, 342 genes were expressed more highly $(\log 2 \geq 2.0)$ in all three somatic embryo samples than in leaf tissues. These genes belong to more than 40 different TF families including $\mathrm{C} 2 \mathrm{H} 2$ (40 genes), AP2EREBP (39), bHLH (31), MYB (26), homeobox (22), WRKY (22), bZIP (13) and MADS-box (12). Several of these TF encoding genes that are important in SE are listed in Table 5. The majority of stress responsive genes that are involved in ethylene responses have been classified in the TF family, AP2-EREBP. In our study we noted that at least 39 genes related to the AP2-EREBP family are more highly expressed in somatic embryos than in leaf tissues. Among those genes, both ethylene responsive TFs, RAP2.6 (AT1G43160) and RAP2.6 L (AT5G13330) were detected at a noticeably higher level in somatic embryos $(\log 2[\mathrm{FC}] \approx 6)$. These two specific TFs play an important role during stress signalling [36]. Additionally, several genes encoding for CYTOKININ RESPONSE FACTORS (CRFs) were also

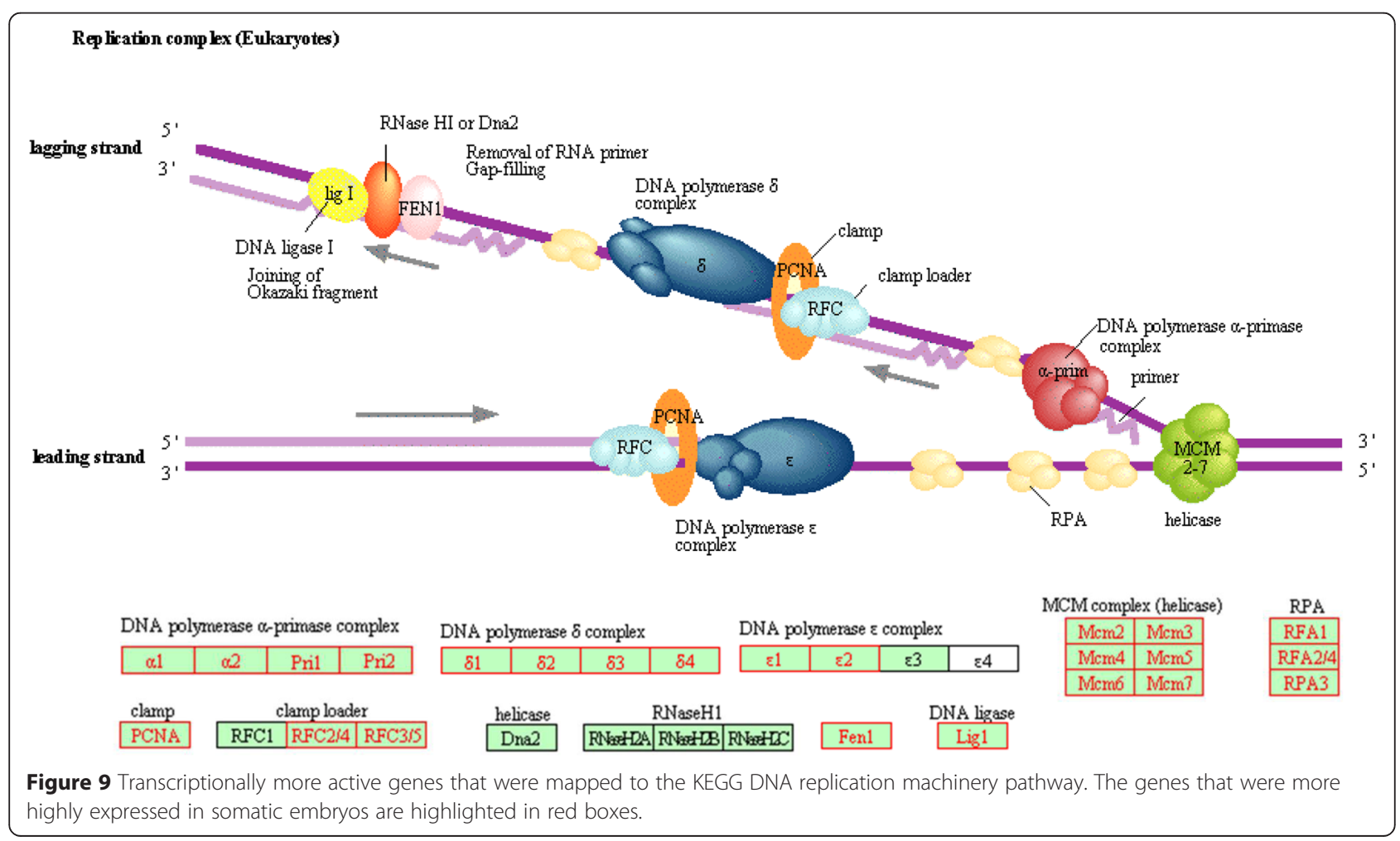




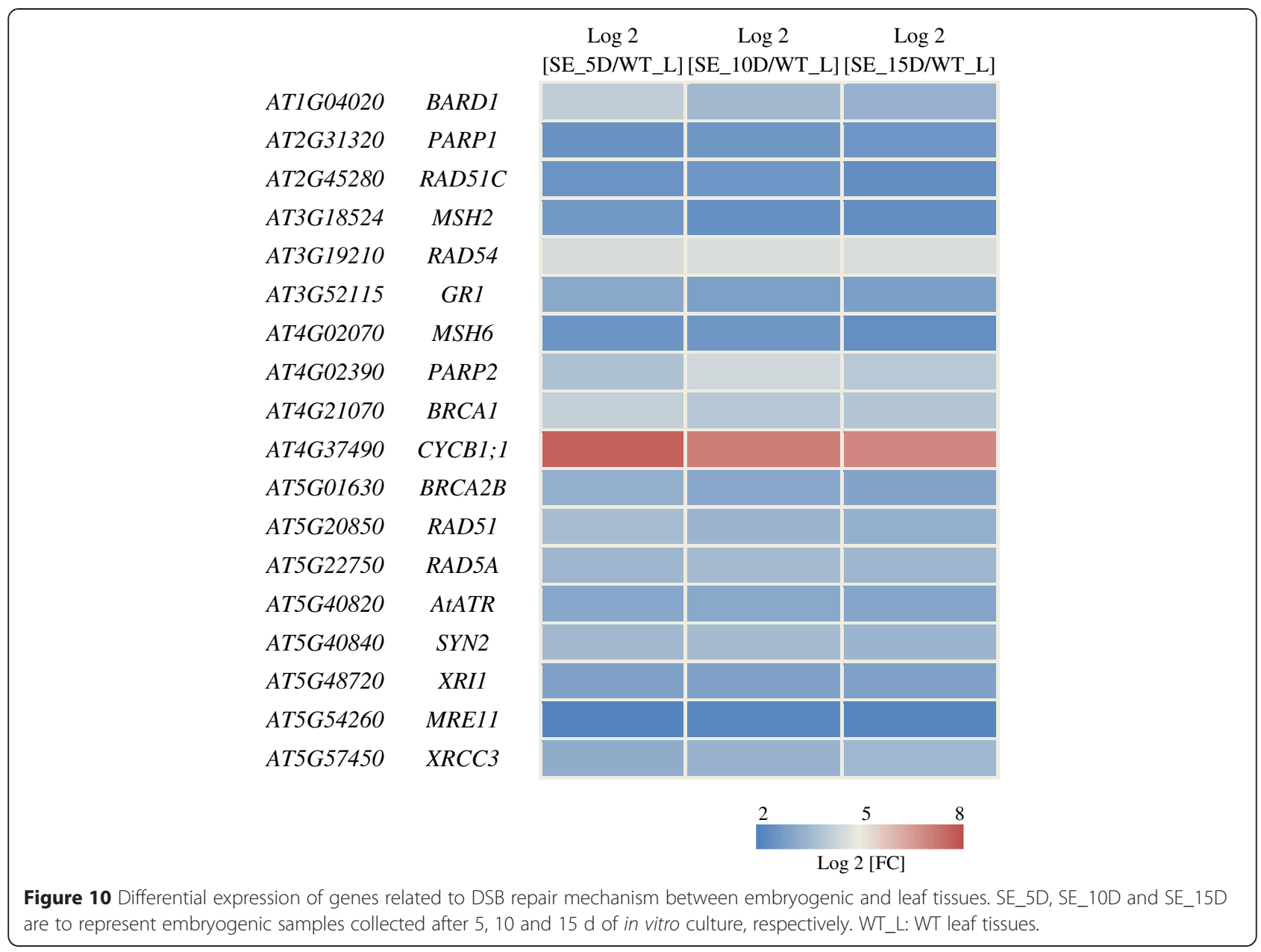

transcriptionally more active in somatic embryos than in leaf tissues i.e. CRF1, CRF2, CRF3, CFR4, CFR5, CRF10 and CRF11. These AP2-domain containing TFs, together with type-B Arabidopsis response regulators, are involved in regulation of embryo, cotyledon and leaf development [37]. Significant expression of RAP2.6, RAP2.6 $L$ and four CRFs (CRF2, CRF3, CRF4 and CRF5) in embryogenic cultures has been reported in a qRT-PCR expression analysis of TFs [38].

Studies so far have discovered several marker genes that regulate somatic-to-embryogenic cell fate in plants [5,39-43]. In order to further validate the expression levels of some of these genes, the transcript levels of 12 well-studied marker genes were filtered from our transcriptome data. As expected none of the marker genes selected is expressed at a high level in leaf tissues (Figure 11). It was evident that FUSCA 3 (FUS3), ABA INSENSITIVE 3 (ABI3), SOMATIC EMBRYOGENESIS RECEPTOR KINASE 1 (SERK1), ATS1 and the LEA genes i.e. ECP31 and ECP63 are expressed at a higher level during early $\mathrm{SE}$, with a gradual reduction during subsequent embryo maturation. Additionally, LEAFY
COTYLEDON 1 (LEC1), WUSCHEL RELATED HOMEOBOX 9 (WOX9) and AGAMOUS-like 15 (AGL15) genes also showed accumulation of more transcripts in early stages as compared to SE_15D; however, the transcripts levels remained relatively stable during 5 and $10 \mathrm{~d}$ after in vitro embryogenesis. Considerable expression of $L E A F Y$ COTYLEDON 2 (LEC2), BABY BOOM (BBM1) and WUSCHEL (WUS) genes was noted in embryos after $10 \mathrm{~d}$ of in vitro culture. Interestingly, the two embryo specific genes, ATS1 and ATS3 [44] showed a similar pattern of transcript accumulation during in vitro embryogenesis where the expression was markedly high in embryos after $5 \mathrm{~d}$ of in vitro culture and then reduced as they develop. In addition, transcripts of both genes were noticeably more abundant in somatic embryos than in WT leaf tissues. Although it has been hypothesized that both of these embryo specific genes may function in maintaining subcellular embryogenic structures and establishing storage material within the cell [44], experimental validation has not yet been reported. Based on our transcript information, it is more likely that both ATS1 and ATS3 genes may have a regulatory role during early SE. 
Table 5 Important TF genes that were more highly expressed in somatic embryos as compared to leaf tissues, ranked in order of TF family

\begin{tabular}{|c|c|c|c|}
\hline Gene ID & Gene short name & TF family & Description \\
\hline AT3G26790 & FUS3 & $\mathrm{ABI3VP1}$ & \multirow[t]{2}{*}{ Regulator of gene expression during late embryogenesis } \\
\hline AT1G28300 & LEC2 & $\mathrm{AB} \mid 3 \bigvee \mathrm{V} 1$ & \\
\hline AT3G24650 & $A B / 3$ & $\mathrm{AB} \mid 3 \bigvee \mathrm{V} 1$ & Essential for seed maturation and a central regulator in ABA signalling \\
\hline AT4G37750 & ANT & AP2-EREBP & Required for control of cell proliferation \\
\hline AT5G57390 & All 5 & AP2-EREBP & $\begin{array}{l}\text { Essential for the developmental transition between the embryonic and } \\
\text { vegetative phases }\end{array}$ \\
\hline AT5G17430 & $B B M$ & AP2-EREBP & Similar to AINTEGUMENTA expressed in embryos \\
\hline AT5G64750 & $A B R 1$ & AP2-EREBP & Involved in ABA signalling \\
\hline AT4G11140 & CRF1 & AP2-EREBP & \multirow{7}{*}{$\begin{array}{l}\text { Members of ERF subfamily; cytokinin response factors function redundantly } \\
\text { to regulate the development of embryos, cotyledons and leaves }\end{array}$} \\
\hline AT1G68550 & CRF10 & AP2-EREBP & \\
\hline AT3G25890 & CRF11 & AP2-EREBP & \\
\hline AT4G23750 & CRF2 & AP2-EREBP & \\
\hline AT5G53290 & CRF3 & AP2-EREBP & \\
\hline AT4G27950 & CRF4 & AP2-EREBP & \\
\hline AT2G46310 & CRF5 & AP2-EREBP & \\
\hline AT3G20840 & PLT1 & AP2-EREBP & \multirow{2}{*}{$\begin{array}{l}\text { A key effector for establishment of the stem cell niche during embryonic } \\
\text { pattern formation }\end{array}$} \\
\hline AT1G51190 & PLT2 & AP2-EREBP & \\
\hline AT1G19850 & MP & ARF & TF (IAA24) mediating embryo axis formation and vascular development \\
\hline AT2G41070 & EEL & bZIP & $\begin{array}{l}\text { Homologous to ABI5; Located in the nucleus and expressed during seed } \\
\text { maturation in the cotyledons and later in the whole embryo }\end{array}$ \\
\hline AT2G36270 & $A B / 5$ & bZIP & $\begin{array}{l}\text { Regulates a subset of LEA genes; involved in ABA signalling during seed } \\
\text { maturation and germination }\end{array}$ \\
\hline AT3G54810 & GATAB & C2C2-Gata & Expressed in the embryo axis and involved in germination \\
\hline AT3G50870 & GATA18 & C2C2-Gata & $\begin{array}{l}\text { A transcriptional regulator required to position the proembryo boundary } \\
\text { in the early embryo }\end{array}$ \\
\hline AT5G07500 & PEl1 & $\mathrm{C} 2 \mathrm{H} 2$ & An embryo-specific zinc finger TF required for heart-stage embryo formation \\
\hline AT5G47670 & $L 1 L$ & CCAAT-HAP3 & A regulator of embryo development \\
\hline AT1G62360 & STM & HOMEOBOX & $\begin{array}{l}\text { Required for shoot apical meristem (SAM) formation during embryogenesis } \\
\text { and for SAM function throughout the lifetime of the plant }\end{array}$ \\
\hline AT5G13790 & AGL15 & MADS & $\begin{array}{l}\text { Preferentially expressed during embryogenesis and key regulator of } \\
\text { embryogenesis }\end{array}$ \\
\hline AT5G53950 & CUC2 & NAC & $\begin{array}{l}\text { With CUC1 redundantly required for embryonic apical meristem formation } \\
\text { and cotyledon separation }\end{array}$ \\
\hline AT3G15170 & CUC1 & NAC & $\begin{array}{l}\text { Involved in shoot apical meristem formation and auxin-mediated lateral } \\
\text { root formation }\end{array}$ \\
\hline AT1G66600 & $A B O 3$ & WRKY & Involved in ABA signalling \\
\hline
\end{tabular}

\section{Expression patterns of genes related to epigenetic modifications}

SE is a highly dynamic developmental process that involves active dedifferentiation of somatic cells, followed by the induction and maturation of embryos. The process of induction of in vitro embryos is mainly regulated by plant growth regulators and stress responses. Apart from that, these factors may contribute to induce epigenetic modifications during SE. Therefore, to gain insight into the expression patterns of genes that regulate these epigenetic signatures, transcript levels of genes related to DNA methylation/demethylation and histone acetylation/deacetylation were studied in-depth. This would provide clues about the level of methylation during in vitro embryogenesis.

Cytosine DNA methylation is one of the well-studied epigenetic modifications in higher plants and is involved in regulation of gene expression and silencing of TEs [45]. In addition, it controls various processes such as embryogenesis and genomic imprinting [46]. This process involves incorporation of a methyl group to the $5^{\prime}$ position of the cytosine base by a group of enzymes called 


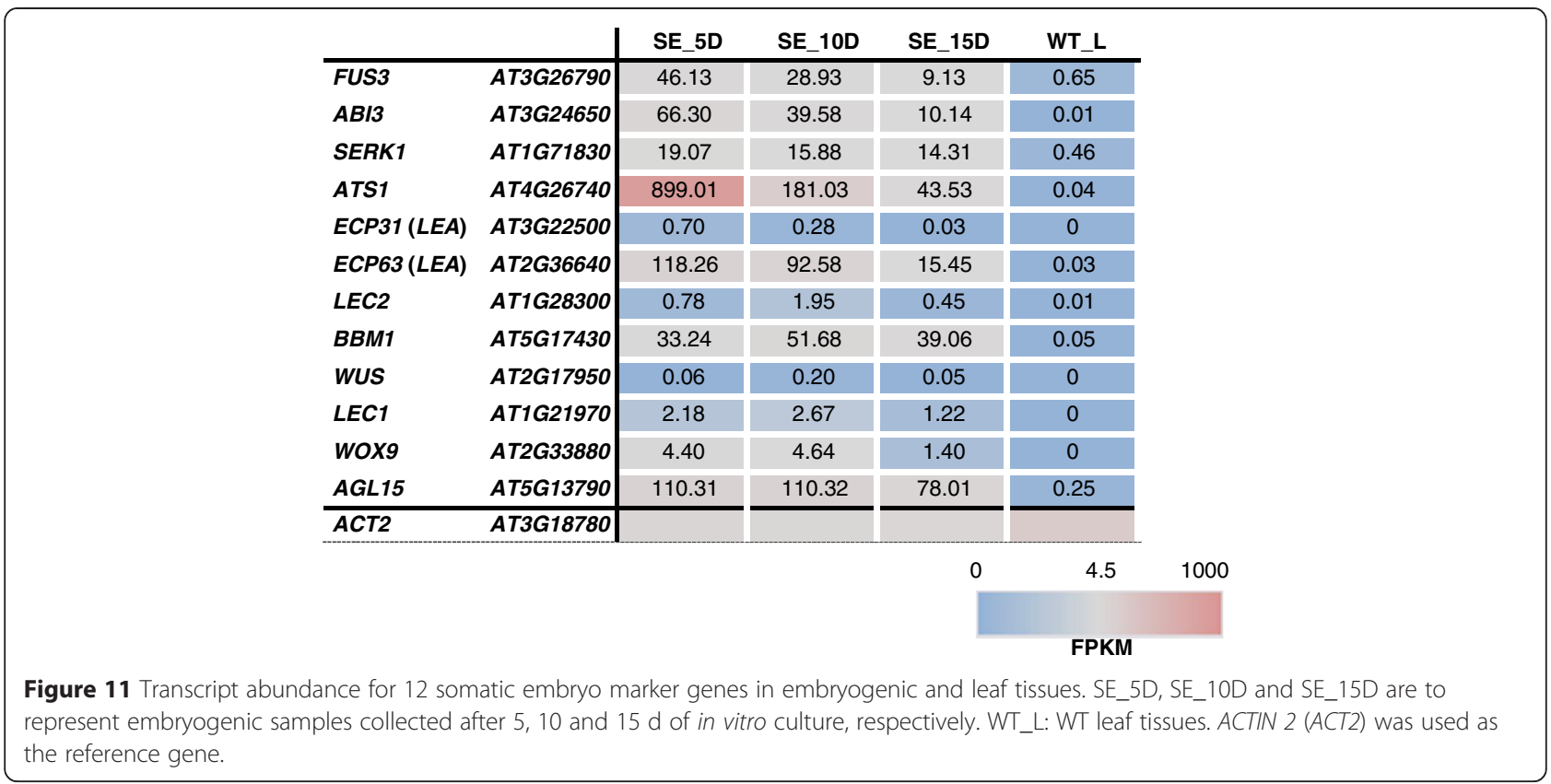

DNA methyltransferases. Although there was no considerable variation in transcript levels of genes related to DNA methylation/demethylation during SE, a marked difference was noticed for these genes between somatic embryo and WT_L transcriptomes (Figure 12; see Additional file 7 for the expression patterns of these genes based on microarray data from Genevestigator). Both METHYLTRANSFERASE 1 (MET1) and CHROMOMETHYLASE 3 (CMT3) genes that play a key role in maintaining cytosine DNA methylation during Arabidopsis embryogenesis [47] were transcriptionally more active in embryogenic tissues (Log2 $[\mathrm{FC}] \geq 2.0)$. In addition, DOMAINS REARRANGED METHYLTRANSFERASE 2 (DRM2), the main de novo methyltransferase found in Arabidopsis [48] also showed accumulation of more transcripts in somatic embryos. The single gene mutants of MET1 (met1-6) in Arabidopsis display developmental defects in ZEs i.e. abnormal cell divisions during early embryogenesis and delayed development as compared to WT embryos [47]. In addition, the reduced expression levels observed for genes that specify cell fate during early embryogenesis (WUSCHEL RELATED HOMEOBOX 2 (WOX2), WOX8 and $Y O D A(Y D A))$ in met1-6 mutants highlights the importance of DNA methylation in maintaining cell identify during early embryogenesis [47]. Likewise, the double mutants of MET1 and CMT3 genes have reduced seed

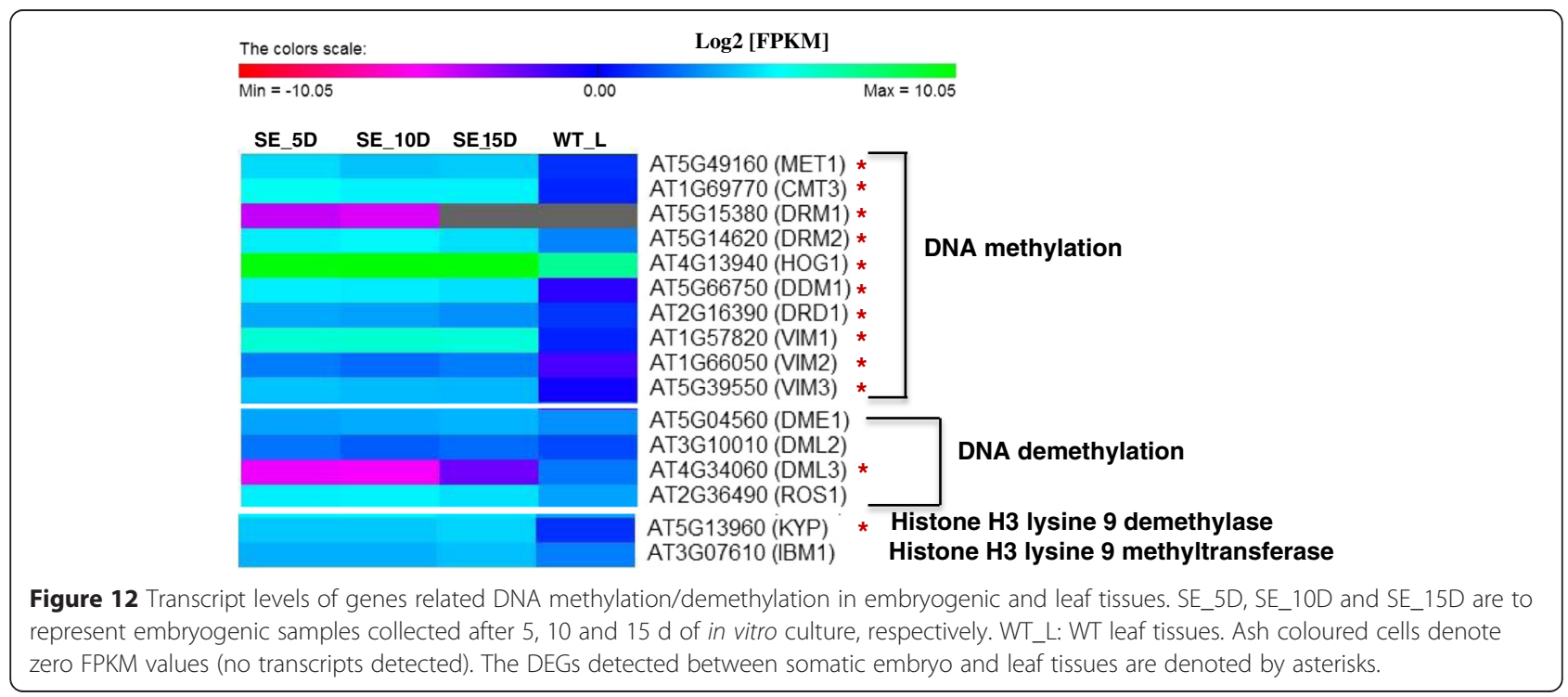


viability. Although further evidence is required, it is believed that DNA methylation may be directly or indirectly involved in the regulation of polar auxin transport during early embryogenesis [47]. Thus, it is possible that the increased expression levels observed for most of the genes related to cytosine DNA methylation in somatic embryos may link to hormone and developmental signalling pathways that take place during in vitro embryogenesis.

Similar to DNA methylation related genes, the histone methylation related gene, KRYTONITE (KYP) also showed higher expression in somatic embryos (Figure 12). This histone methyltransferase, KYP functions together with CMT3 to induce and maintain CHG trinucleotide methylation in TEs $[46,49]$. Increased accumulation of both CMT3 and KYP transcripts in somatic embryos than in leaf tissues ( $\log 2[F C] \geq 2.0)$ suggests activation of CMT3 - KYP mediated cytosine methylation during SE to inactivate mobile transposons.

Furthermore, the proteins that bind to methylated cytosine also play a key crucial role in maintaining epigenetic status. Of these, three VARIANT IN METHYLATION (VIM) proteins have been identified as predominant regulators that maintain global CG dinucleotide methylation patterns and gene silencing in Arabidopsis [50]. Expression analysis of VIM encoding genes through RT-PCR reports higher transcript levels for VIM1 and VIM2 in Arabidopsis inflorescences than in leaves. Moreover, a relatively similar level of gene expression has been detected in both inflorescences and leaves for VIM3. However, we found that all three VIM encoding genes are expressed at a considerably higher level in somatic embryos than in leaf tissues ( $\log 2[\mathrm{FC}] \geq 2.0)$. Of these, VIM1 showed the highest transcript abundance in embryogenic tissues; this suggests VIMs may play a role in maintaining methylation patterns during embryogenesis in vitro.

In addition to DNA methylation, DNA demethylation is also equally important in regulation of gene expression patterns during developmental processes. In Arabidopsis, three genes related to the DME family (DEMETER 1 (DME1), DEMETER-LIKE 2 (DML2) and DEMETER-LIKE 3 (DML3)) and a homolog of DME known as REPRESSOR OF SILENCING 1 (ROS1), that contain a DNA glycosylase domain, are involved in DNA demethylation [46]. It was evident that with the exception of $D M L 3$, other genes related to DNA demethylation were expressed at a relatively similar level in somatic embryos and WT leaf tissues. However, DML3 that catalyses active removal of 5-methylcytosine showed considerably lower expression in somatic embryos as compared to WT_L with an average $\log 2$ ratio of -6 . It is reported that although $D M L 3$ is expressed in siliques and leaves, it is not expressed in mature seeds. In addition, the mRNA stability of $D M L 3$ may depend on the plant tissue type [51]. Thus, based on our expression data, it can be assumed that $D M L 3$ transcripts are more stable in leaves as compared to embryos.

Histone acetylation/deacetylation is another important post translational histone modification that regulates gene expression patterns during plant development as well as in response to various environmental conditions [52]. In general, hyperacetylation activates transcription and mediated by a group of enzymes known as histone acetyltransferases (HATs). The present study found that with the exception of the CBP family member $H A C 2$, other HATs are expressed stably during SE. The $H A C 2$ gene was expressed at a considerably higher level in later stages (SE_10D and SE_15D) as compared to SE_5D. In addition, the differential gene expression analysis of HATs between embryogenic and leaf tissues showed noticeable accumulation of $\mathrm{HAC} 2$ transcript in somatic embryos than in WT_L. However, to date no histone acetylation activity has been reported for this gene in Arabidopsis [53]. In animals, CBP family proteins are considered as active transcriptional co-activators that regulate processes such as cell cycle and cell differentiation. Moreover, CBP family members have been identified as essential regulators of mouse embryogenesis and pattern formation [54]. Therefore, detection of increased expression of $H A C 2$ gene in somatic embryos suggests that it is likely to function during SE, which also involves active cell divisions. In addition to $H A C 2$, two genes from the GNAT family, HAG2 and HAG3 also showed accumulation of more transcripts in somatic embryos than in leaf tissues with an average $\log 2[\mathrm{FC}]$ of 2.0. However, the majority of plant HATs have been identified by sequence characterization to other eukaryotes; thus, the functional characterization of the majority of these genes remains largely unknown. Some of the functionally characterized HATs include HAG1, HAF2, HAC1, HAC5 and HAC12 [55]. For instance, a study on plant $H A C 1$ has shown its function in reproductive and vegetative development in Arabidopsis [56]; T-DNA mutants of HAC1 have shown reduced fertility with shorter siliques as compared to WT plants and late flowering phenotypes [57]. Therefore, it is possible that HAG2, HAG3 and $H A C 2$ may also have a function in embryogenesis.

On the other hand, histone deacetylation/hypoacetylation may lead to a transcriptional repression. This process is catalysed by histone deacetylases (HDACs) $[45,58]$. Expression profile analysis of 18 HDACs comprising 12 from the RPD3 family, four from the plant specific HD-tuins family and two from the Sirtuin HDAC family showed that with the exception of $H D A 7$, all the $H D A C$ s are expressed throughout the SE in a relatively stable manner. Transcripts for the HDA7 gene were only detected in SE_10D. Interestingly, it was noted that all four HD-tuins family members expressed at a relatively similar level in somatic embryos and shared a similar expression pattern 
during in vitro embryo development. A similar pattern of gene expression has been observed for HD-tuins family members in somatic embryos and seed derived embryos through in situ hybridization, indicating a functional redundancy of those genes in plant development [58]. Additionally, these genes were transcriptionally more active in embryogenic tissues than in leaf tissues. Similarly, Zhou et al. [59] report accumulation of HDT1, HDT2 and HDT3 transcripts in somatic embryos derived from transgenic Arabidopsis lines that over express BBM gene. However, it was not clear, whether the accumulation of HD-tuins type genes is due to formation of somatic embryos or overexpression of $B B M$ in transgenic lines that exhibit a higher frequency of induced embryos [59]. The expression information in the present study provides strong support for the suggestion that HD-tuins family HDACs are important in Arabidopsis SE and are more likely to regulate histone modifications in target genes to maintain a high methylation status during SE. However, additional information is needed to determine whether the increased transcript levels observed for HD-tuins in somatic embryos has a link to hormone or developmental signalling pathways that take place during embryogenesis.

In general, detection of increased transcript levels for key genes involved in DNA methylation and histone deacetylation provides an indirect indication of increased epigenetic modifications during in vitro embryogenesis. However, it is not clear whether the expression changes we observed are due to in vitro conditions (i.e. externally supplied auxin, stress responses) or increased epigenetic signatures. Therefore, it would be valuable to conduct a global analysis of the epigenome architecture of somatic embryos in order to understand the expression patterns of diverse embryogenesis related genes.

\section{In silico expression analysis to identify genes more likely to be involved in SE}

Identification of genes more likely to be involved in somatic embryo development would help in the discovery of novel molecular markers for future embryogenesis studies. As a preliminary study, to obtain an estimate of the overlap between genes expressed in somatic embryos and actively dividing callus, 100 genes were randomly selected from our list of genes that showed a higher level of expression in somatic embryos than in non-dividing leaf tissues (a total of $(4,951)$ ). It was considered likely that the majority of genes highly expressed in somatic embryos could also be expressed at a higher level in actively dividing cells, for example in callus. In order to address this possibility a comparison of expression data from somatic embryos, callus and leaf was conducted. This concluded that approximately 5\% of genes are expressed at a higher level in somatic embryos than in actively dividing callus or non-dividing leaf tissues ( $\log 2$
$[\mathrm{FC}] \geq 2.0$ ) (See Additional file 8 for the clustering analysis of microarray expression data from somatic embryos (original repository: Gene Expression Omnibus (GEO (GSE17610); experiment ID: AT-00508), callus (original repository: GEO (GSE8994); experiment ID: AT-00265) and leaf tissues (original repository: GEO (GSE8994); experiment ID: AT-00265). These data were filtered for the randomly selected gene list (100 genes)).

In summary, comparison of microarray gene expression data for somatic embryos (AT-00508) and callus (AT-00265) tissues identified a minimum set of 158 genes that are more highly expressed in somatic embryos than in actively dividing callus cells. Furthermore, subsequent hierarchical clustering of expression data for these 158 genes in samples: somatic embryos (AT-00508), callus (AT-00265), leaf (AT-00265) and torpedo stage zygotic embryos (original repository: GEO (GSE47884); experiment ID: AT-00629) exhibited a distinct cluster with 49 genes (Figure 13) having a comparatively higher level of expression in somatic embryos than in torpedo stage zygotic embryos (See Additional file 9 for the gene description). Furthermore, expression analysis of these genes in other anatomical parts using the Genevestigator tool showed the majority of these genes have a distinct pattern of expression in somatic embryos as compared to other anatomical parts compared (Figure 14). Therefore, these genes could be considered as potential candidate genes for future in vitro embryogenesis related studies.

Interestingly, this subset of 49 genes included several genes responsible for oxidative stress such as AT1G68850, AT2G18980, AT5G14130, AT5G17820 and AT5G44380; genes responsible for salt stress, AT4G02330 and AT4G27400; genes encoding for LEA proteins, AT3G19430, AT4G27400, AT5G54370 and AT5G60530; genes involved in auxin biosynthesis, AT4G28720 and AT5G51470. Moreover, further analysis of these genes revealed the four genes encoding for LEA proteins are positively coexpressed (Pearson's correlation coefficient $>0.60$ ) with both the auxin biosynthesis genes, AT4G28720 and AT5G51470 (Additional file 10). Although these genes were expressed at a higher level in somatic embryos than in other tissues, further studies are recommended to speculate whether these genes are differentially expressed during SE or in response to exogenously supplied auxin, 2, 4-dichlorophenoxyacetic acid (2, 4-D).

\section{Conclusions}

In summary, this study provides a high resolution transcriptome dataset for Arabidopsis SE. A comparative analysis of expression profiles between different somatic embryo developmental time-points as well as between embryogenic and leaf tissues provided subsets of DEGs. Functional characterization of those gene subsets based 


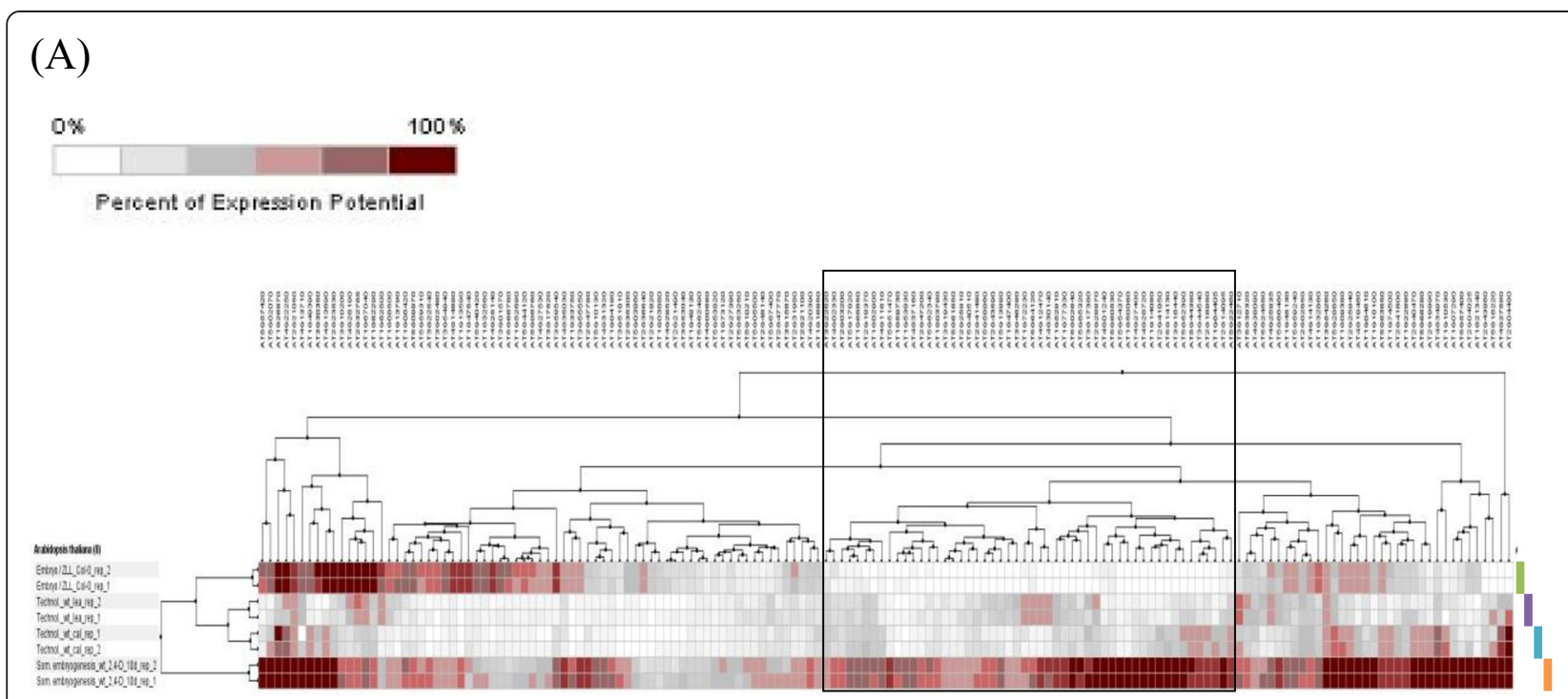

\section{(B)}
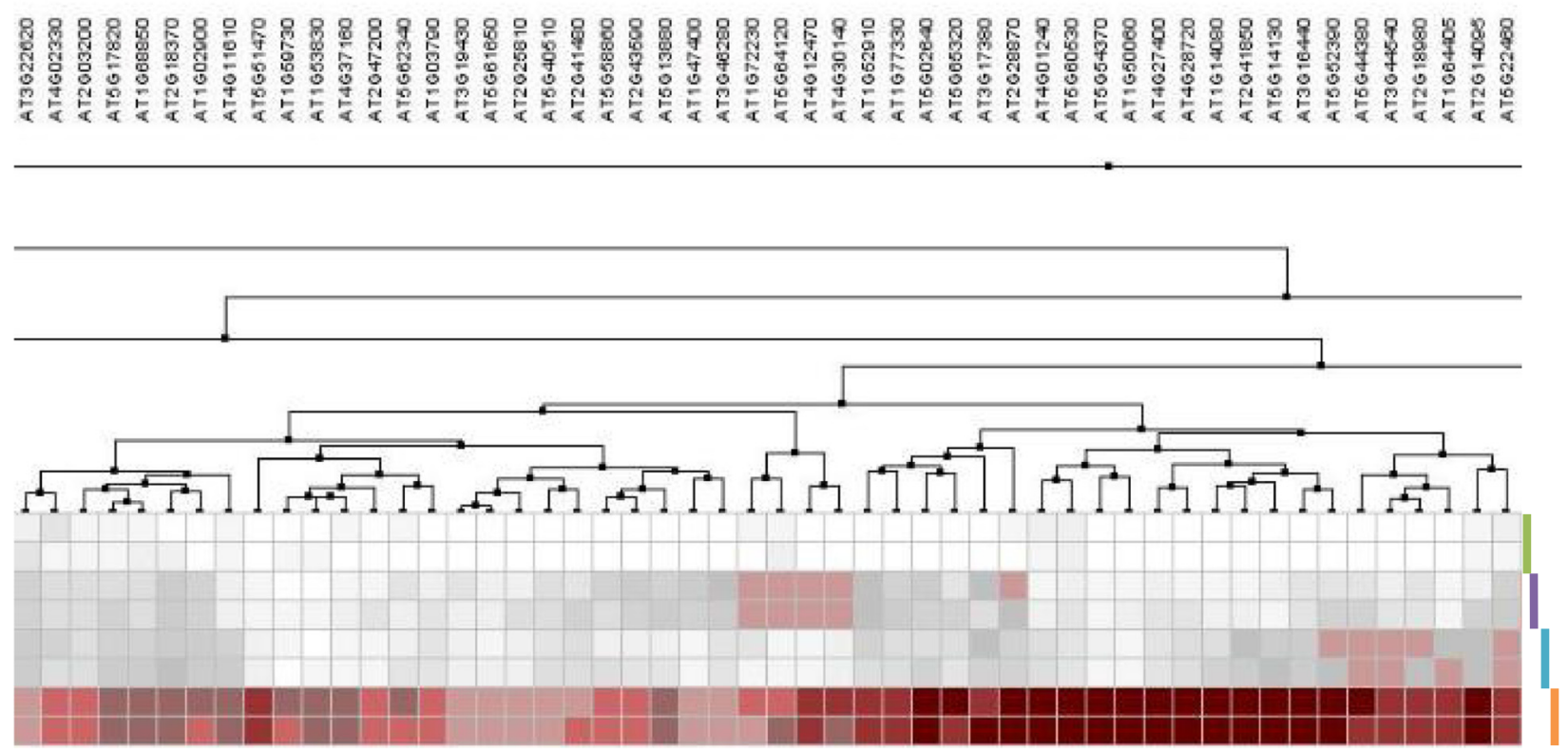

Figure 13 Hierarchical clustering (using Euclidian distance measure) for a list of 158 genes expressed at a higher level in somatic embryos (AT-00508) than in callus (AT-00265), torpedo stage zygotic embryos (AT-00629) or leaf tissues (AT-00265). (A) Hierarchical clustering of 158 genes; (B) Expansion of the clade that showed a higher level of expression in somatic embryos than in callus, torpedo stage embryos or leaf tissues. Green, purple, blue and orange lines represent expression data for torpedo stage zygotic embryos, leaf tissues, callus and somatic embryos, respectively. The Genevestigator hierarchical clustering tool was used to construct the clustering tree.

on significantly enriched GO terms and over-represented biological pathways is presented here. However, further functional characterization and validation of these genes at a molecular level is recommended. Interestingly, the comparison of somatic embryo and leaf tissue derived transcriptomes revealed distinct expression profiles for the majority of genes involved in cell cycle, DNA replication and repair mechanism, DNA cytosine methylation and histone deacetylation. The latter results suggest a particular role for epigenetic regulation in the SE process, and may provide useful leads in the design of systems for the improved induction of embryonic cells from somatic tissue. Interestingly, in silico expression analysis of genes using publicly available microarray expression data identified a subset of 49 genes that are more likely to be involved in SE, and could be considered as potential 


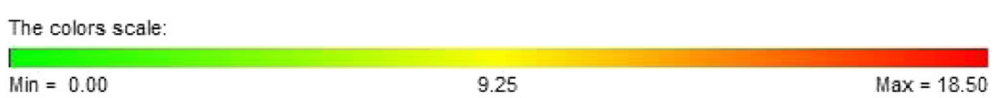

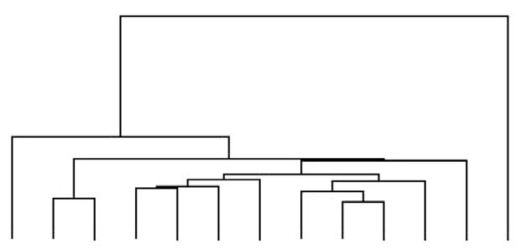

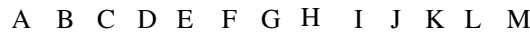

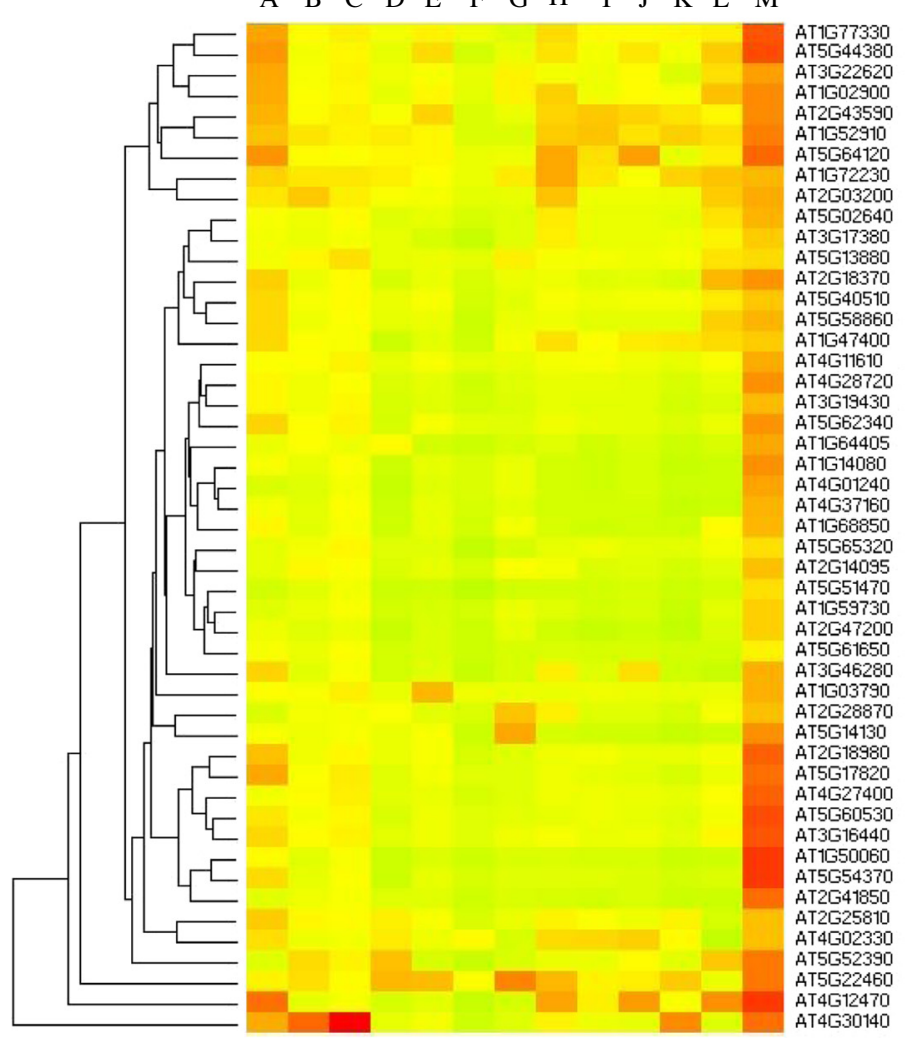

Figure 14 Expression pattern 49 genes expressed at a higher level in somatic embryos than in callus, torpedo stage zygotic embryos or leaf tissues in a range of anatomical parts. A: seedling; B: stamen; C: pollen; D: pistil; E: seed; F: embryo; G: testa; H: inflorescence stem; I: juvenile leaf; J: adult leaf; K: shoot apex; L: hypocotyl; M: somatic embryos. Expression data represent average log 2 signal values for replicates of each

tissue sample.

candidate genes for future embryogenesis studies in vitro. In conclusion, the transcriptomic data generated in the present study for Arabidopsis somatic embryos provides insights into future functional studies and into the development of gene regulatory networks for model species, as a means of understanding the molecular mechanisms that control SE.

\section{Methods}

\section{Plant material and growth conditions}

Seeds of the $A$. thaliana Columbia ecotype (accession number: N70000) were supplied by the Nottingham Arabidopsis Stock Centre, UK. Mother plants were grown on vermiculite containing soil at $25^{\circ} \mathrm{C}$ in $60 \%$ relative humidity with a photoperiod of $16 \mathrm{~h}$ under controlled environmental conditions in Fitotron plant growth chambers (Weiss Gallenkamp, UK).

The direct somatic embryo induction method described by Gaj [60] was used to induce somatic embryos from WT Arabidopsis. In brief, immature zygotic embryos (IZEs), dissected from green siliques of 6-to-8 week old plants, containing embryos at the early bent-cotyledonary stage were used as explants. Initially, all the siliques were surface sterilized by a $10 \mathrm{~min}$ incubation in a commercial bleach $\left(\right.$ Domestos $^{\mathrm{TM}}$ ) containing 20 drops of Tween-20 ${ }^{\circ}$ (BDH Laboratory supplies, UK) per $100 \mathrm{ml}$. After rinsing 
in sterile water for three times, IZEs (approximately $400-500 \mu \mathrm{m}$ in size) were excised by opening surface sterilized siliques, under a dissecting microscope (Stemi SR, Zeiss, Germany), and used to induce somatic embryos. Ten IZEs were inoculated on each Petri dish $(90 \mathrm{~mm})$ containing approximately $20 \mathrm{ml}$ of autoclaved basal Gamborg's B5 medium (containing vitamins; Duchefa Biochemie, The Netherlands) supplemented with $20.0 \mathrm{~g} / \mathrm{L}$ sucrose (BDH Laboratory supplies, UK), $3.5 \mathrm{~g} / \mathrm{L}$ Phytagel (Sigma-Aldrich, USA), $5.0 \mu \mathrm{M}$ of 2, 4-D (Sigma-Aldrich, USA) at pH $5.8 \pm 0.01$. The cultures were then incubated at $24^{\circ} \mathrm{C}$ with a photoperiod of $16 \mathrm{~h}$, under white fluorescent light $\left(90 \mu \mathrm{mol} \mathrm{m}{ }^{-2} \mathrm{~s}^{-1}\right.$ intensity).

\section{Tissue sampling and RNA extraction}

For RNA extraction and subsequent transcriptome sequencing, somatic embryos were collected at three distinct time-points after initial inoculation, $5 \mathrm{~d}$ (SE_5D), $10 \mathrm{~d}$ (SE_10D) and $15 \mathrm{~d}$ (SE_15D) (Additional file 11). Approximately 700 somatic embryos induced at each timepoint were pooled together to obtain a sufficient quantity of starting material for RNA extraction. In addition, two mature leaves collected at the time of flowering from WT plants (WT_L) were pooled to extract RNA. Total RNA was extracted using the RNeasy ${ }^{\circ}$ Plant Mini Kit (Qiagen, UK) according to the manufacturer's instructions and treated with DNase I (Qiagen, UK) to remove any contaminated DNA. The eluted total RNA was quantified using a BioAnalyzer 2100 (Agilent Technologies, CA) by Source Bioscience, UK.

\section{cDNA library construction and Illumina RNA-Seq}

The cDNA libraries were constructed and sequenced by Source Bioscience, UK in accordance with the Illumina TruSeq RNA sample preparation guide v2 for Illumina paired-end multiplexed sequencing. In brief, the poly-AmRNA in the extracted total RNA samples was purified using Illumina poly-T oligo-attached magnetic beads in two rounds of purification steps according to the manufacturer's instruction. During the second step of poly-A RNA elution, the mRNA was fragmented and primed with random hexamers for cDNA synthesis. The first strand cDNA was synthesized from fragmented mRNA using reverse transcriptase and random primers. In a subsequent step, the RNA template was removed and a replacement was synthesized to construct double-stranded
cDNA. After double stranded cDNA synthesis, ends were repaired and an A-base was added to the blunt end fragments. Thereafter, Illumina indexing adapters (Table 6) were ligated according to the standard protocol for pooling of samples prior to sequencing and for subsequent identification of pooled samples in downstream analysis. The cDNA fragments that have adapter molecules on both ends were subjected to 15 rounds of PCR amplification. The concentration and size distribution of the synthesized cDNA libraries were confirmed using an Agilent BioAnalyzer 2100. The successfully amplified and indexed libraries were pooled prior to sequencing (two samples per lane). The resulted pools were diluted to $10 \mathrm{nM}$. The molarity and size distribution were confirmed using an Agilent BioAnalyzer 2100. Finally, pooled samples were loaded at a concentration of $8 \mathrm{pM}$ into each lane of an Illumina HiSeq 2000 flow cell v3 and sequenced with 50 bp paired-end reads.

\section{Mapping sequence reads to the Arabidopsis reference genome}

Pre-built TAIR10 index was downloaded from the Illumina iGenomes webpage (http://support.illumina.com/sequencing/ sequencing_software/igenome.html; Arabidopsis_thaliana_Ensembl_TAIR10.tar.gz) and used as the reference for mapping. Quality of each raw sequence data file was analysed using open-source software FastQC (http://www.bioinformatics. babraham.ac.uk/projects/fastqc/). After removing adapter sequences, the high quality sequence reads were aligned and assembled to the $A$. thaliana reference genome (TAIR10) using TopHat 2.0.6 (http://ccb.jhu.edu/software/tophat/index. shtml) with Bowtie 2 (http://bowtie-bio.sourceforge.net/index. shtml) with the default parameters, allowing only 2 base mismatches per read alignment [61]. Aligned sequence reads were visualized using the IGV version 2.2 (http:// www.broadinstitute.org/igv/) and Integrated Genome Browser version 7.0.4 (IGB) (http://bioviz.org/igb/). The general statistics of mapped reads were acquired using the Sequence Alignment/Map (SAM) tool (http://samtools. sourceforge.net/).

\section{Normalization of transcript levels of expressed genes}

The assembled transcripts were merged with the Arabidopsis reference annotation downloaded from the Illumina iGenome (Arabidopsis_thaliana_Ensembl_TAIR10.tar.gz)

Table 6 True Seq adapter sequences

\begin{tabular}{|c|c|}
\hline Sample ID & Adapter sequence $\left(5^{\prime}->3^{\prime}\right)$ \\
\hline SE_5D & GATCGGAAGAGCACACGTCTGAACTCCAGTCACATCACGATCTCGTATGCCGTCTTCTGCTTG \\
\hline SE_10D & GATCGGAAGAGCACACGTCTGAACTCCAGTCACCGATGTATCTCGTATGCCGTCTTCTGCTTG \\
\hline SE_15D & GATCGGAAGAGCACACGTCTGAACTCCAGTCACTTAGGCATCTCGTATGCCGTCTTCTGCTTG \\
\hline WT_L & GATCGGAAGAGCACACGTCTGAACTCCAGTCACTGACCAATCTCGTATGCCGTCTTCTGCTTG \\
\hline
\end{tabular}


and the transcript abundance were estimated in terms of FPKM.

\section{Analysis of differentially expressed genes (DEGs) and downstream bioinformatic analysis}

The FC was calculated as a ratio of transcript levels (FPKM) between different somatic embryo developmental time-points or between different tissue types (embryogenic vs. leaf). Genes with $-2.0 \geq \log 2[\mathrm{FC}] \geq 2.0$ were considered as DEGs. K-mean clustering on these gene subsets was performed using the MultiExperiment Viewer $(\mathrm{MeV})$ software with 10 user defined clusters (http://www. tm4.org/).

The PLAZA web tool (http://bioinformatics.psb.ugent. be/plaza/) [62] was used to identify significantly enriched GO terms within a given subset of genes. In addition, functional networks based on enriched biological processes related GO terms were generated using Cytoscape (http:// www.cytoscape.org/) software with the ClueGO plugin [63]. The SkyPainter tool in the Arabidopsis Reactome was used to identify the most common plant biological pathways (http://arabidopsisreactome.org/userguide/userguide.html) that exist within the subsets of DEGs.

Transcript levels of genes related to DNA replication and repair mechanisms [34], cell cycle [27,28], methylation/demethylation [46] and histone acetylation/deacetylation [57] were filtered from our transcriptome data to provide a comprehensive overview of their gene expression patterns during in vitro embryogenesis.

\section{In silico validation of gene expression patterns}

To validate the gene expression patterns, Affymetrix microarray expression data were filtered for somatic embryos, zygotic embryos, callus and leaf tissues, and used to produce expression heat maps using the Genevestigator software tool (https://genevestigator.com/gv/plant.jsp).

\section{Availability of supporting data}

The data set(s) supporting the results of this article are available in the European Bioinformatics Institute ArrayExpress repository, E-MTAB-2403 and E-MTAB2465 (www.ebi.ac.uk/arrayexpress/).

\section{Additional files}

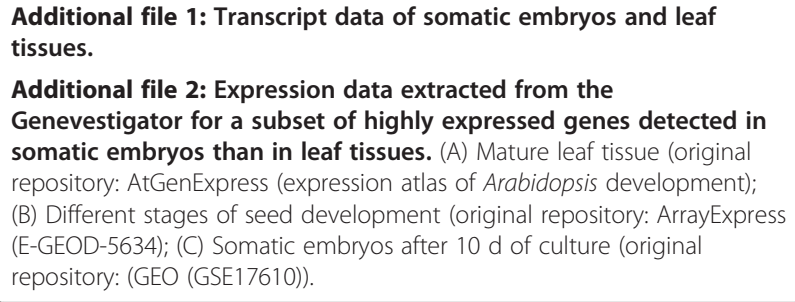

Additional file 3: List of potential candidate genes for molecular studies. This subset of genes was prepared from a list of more highly expressed genes detected in somatic embryos in the present study which had links to known mutant seed phenotypes (SeedGenes database).

Additional file 4: Expression data extracted from the Genevestigator for a subset of highly expressed genes detected in WT leaf than in somatic embryos. (A) Mature leaf tissue (original repository: AtGenExpress (expression atlas of Arabidopsis development); (B) Different stages of seed development (original repository: ArrayExpress (E-GEOD-5634); (C) Somatic embryos after $10 \mathrm{~d}$ of culture (original repository: GEO (GSE17610)).

Additional file 5: Expression data extracted from the Genevestigator for a subset of genes related to cell cycle. (A) Mature leaf tissue (original repository: AtGenExpress (expression atlas of Arabidopsis development); (B) Different stages of seed development (original repository: ArrayExpress (E-GEOD-5634); (C) Somatic embryos after $10 \mathrm{~d}$ of culture (original repository: GEO (GSE17610))

Additional file 6: Expression data extracted from the Genevestigator for a subset of genes related to DNA replication. (A) Mature leaf tissue (original repository: AtGenExpress (expression atlas of Arabidopsis development); (B) Different stages of seed development (original repository: ArrayExpress (E-GEOD-5634); (C) Somatic embryos after $10 \mathrm{~d}$ of culture (original repository: GEO (GSE17610)).

Additional file 7: Expression data extracted from the Genevestigator for a subset of genes related to DNA cytosine methylation. (A) Mature leaf tissue (original repository: AtGenExpress (expression atlas of Arabidopsis development); (B) Different stages of seed development (original repository: ArrayExpress (E-GEOD-5634); (C) Somatic embryos after $10 \mathrm{~d}$ of culture (original repository: GEO (GSE17610)).

Additional file 8: Hierarchical clustering (using Euclidian distance measure) for a subset of 100 randomly selected genes, using microarray expression data from somatic embryos (AT-00508), callus (AT-00265) and leaf (AT-00265) in Arabidopsis. The gene list employed in this study was selected from the list of more highly expressed genes identified in somatic embryos in the present study. Genevestigator hierarchical clustering tool was used to construct the clustering tree. The genes more highly expressed in somatic embryos as compared to leaf and callus tissues are highlighted in yellow (Log2 [FC] $\geq 2.0$ ). Orange, blue and purple colours highlight the samples from somatic embryos, callus and leaf tissues, respectively.

Additional file 9: Description of the $\mathbf{4 9}$ genes that exhibited a higher level of expression in somatic embryos (AT-00508) than in callus (AT-00265), torpedo stage zygotic embryos (AT-00629) or leaf tissues (AT-00265), based on microarray data extracted from the Genevestigator tool.

Additional file 10: List of the top two hundred genes most highly co-expressed with AT4G28720 and AT5G51470. Co-expression data generated using the Genevestigator co-expression analysis tool, and based on 72 different tissue samples.

Additional file 11: Time-points selected for tissue sampling and total RNA extraction from IZE explants cultured on B5 medium supplemented with $5 \mu$ M 2, 4-D. Scale bars $=200 \mu \mathrm{m}$.

\section{Abbreviations}

2,4-D: 2, 4-dichlorophenoxyacetic acid; bHLH: Basic helix-loop-helix; BP: Biological processes; CC: Cellular components; DEG: Differentially expressed gene; FC: Fold change; FPKM: Fragments Per Kilobase of exon per Million fragments mapped; GEO: Gene Expression Omnibus; GO: Gene Ontology; IZE: Immature zygotic embryo; MF: Molecular function; RNA-Seq: RNA sequencing; SE: Somatic embryogenesis; SE_5D: Transcriptome of embryogenic cultures after $5 \mathrm{~d}$ of inoculation; SE_10D: Transcriptome of embryogenic cultures after $10 \mathrm{~d}$ of inoculation; SE_15D: Transcriptome of embryogenic cultures after $15 \mathrm{~d}$ of inoculation; TE: Transposable element; TF: Transcription factor; WT: Wild type; WT_L: Transcriptome of WT leaf tissues; ZE: Zygotic embryo.

\section{Competing interests}

The authors declare that they have no competing interests. 


\section{Authors' contributions}

JMD conceived of the study, and participated in the design of the study and helped to draft the manuscript. AMW participated in the design of the study, performed experiments, analysis and interpretation of data, and drafted the manuscript. All authors read and approved the final manuscript.

\section{Acknowledgements}

This project was funded by The University of Reading, U.K. The design, collection, analysis, and interpretation of data; the writing of the manuscript; and the decision to submit the manuscript for publication were the sole responsibility of the authors.

Received: 5 August 2014 Accepted: 30 March 2015

Published online: 16 April 2015

\section{References}

1. Zimmerman JL. Somatic embryogenesis: a model for early development in higher plants. Plant Cell. 1993;5:1411-23.

2. Quiroz-Figueroa ER, Rojas-Herrera R, Galaz-Avalos RM, Loyola-Vargas VM. Embryo production through somatic embryogenesis can be used to study cell differentiation in plants. Plant Cell Tiss Organ Cult. 2006;86:285-301.

3. Yang $X$, Zhang X. Regulation of somatic embryogenesis in higher plants. Crit Rev Plant Sci. 2010:29:36-57.

4. Smertenko A, Bozhkov P. The life and death signalling underlying cell fate determination during somatic embryogenesis. In: Nick P, Opatrny Z, editors. Applied plant cell biology. Volume 22. Berlin, Heidelberg: Springer-Verlag; 2014. p. 131-78.

5. Gaj MD, Zhang S, Harada JJ, Lemaux PG. Leafy cotyledon genes are essential for induction of somatic embryogenesis of Arabidopsis. Planta. 2005:222:977-88.

6. Ledwon A, Gaj MD. LEAFY COTYLEDON2 gene expression and auxin treatment in relation to embryogenic capacity of Arabidopsis somatic cells. Plant Cell Rep. 2009;28:1677-88.

7. Zeng F, Zhang X, Zhu L, Tu L, Guo X, Nie Y. Isolation and characterization of genes associated to cotton somatic embryogenesis by suppression subtractive hybridization and macroarray. Plant Mol Biol. 2006;60:167-83.

8. Domoki M, Gyorgyey J, Biro J, Pasternak TP, Zvara A, Bottka S, et al. Identification and characterization of genes associated with the induction of embryogenic competence in leaf-protoplast-derived alfalfa cells. Biochim Biophys Acta. 2006;1759:543-51.

9. Cairney J, Pullman GS. The cellular and molecular biology of conifer embryogenesis. New Phytol. 2007;176:511-36.

10. Sharma SK, Millam S, Hein I, Bryan GJ. Cloning and molecular characterisation of a potato SERK gene transcriptionally induced during initiation of somatic embryogenesis. Planta. 2008;228:319-30.

11. Thibaud-Nissen F, Shealy RT, Khanna A, Vodkin LO. Clustering of microarray data reveals transcript patterns associated with somatic embryogenesis in soybean. Plant Physiol. 2003;132:118-36.

12. Lin HC, Morcillo F, Dussert S, Tranchant-Dubreuil C, Tregear JW, Tranbarger TJ. Transcriptome analysis during somatic embryogenesis of the tropical monocot Elaeis guineensis: evidence for conserved gene functions in early development. Plant Mol Biol. 2009;70:173-92.

13. Low ET, Alias H, Boon SH, Shariff EM, Tan CY, Ooi LC, et al. Oil palm (Elaeis guineensis Jacq.) tissue culture ESTs: identifying genes associated with callogenesis and embryogenesis. BMC Plant Biol. 2008;8:62.

14. Che P, Love TM, Frame BR, Wang K, Carriquiry AL, Howell SH. Gene expression patterns during somatic embryo development and germination in maize Hi II callus cultures. Plant Mol Biol. 2006;62:1-14.

15. Lippert D, Zhuang J, Ralph S, Ellis DE, Gilbert M, Olafson R, et al. Proteome analysis of early somatic embryogenesis in Picea glauca. Proteomics. 2005;5:461-73

16. Li Q, Zhang S, Wang J. Transcriptome analysis of callus from Picea balfouriana. BMC Genomics. 2014;15:553.

17. Marsoni M, Bracale M, Espen L, Prinsi B, Negri AS, Vannini C. Proteomic analysis of somatic embryogenesis in Vitis vinifera. Plant Cell Rep. 2008;27:347-56.

18. Imin N, Nizamidin M, Daniher D, Nolan KE, Rose RJ, Rolfe BG. Proteomic analysis of somatic embryogenesis in Medicago truncatula explant cultures grown under 6-benzylaminopurine and 1-naphthaleneacetic acid treatments. Plant Physiol. 2005;137:1250-60.
19. Li K, Zhu W, Zeng K, Zhang Z, Ye J, Ou W, et al. Proteome characterization of cassava (Manihot esculenta Crantz) somatic embryos, plantlets and tuberous roots. Proteome Sci. 2010;8:10

20. Baba Al, Nogueira FCS, Pinheiro CB, Brasil JN, Jereissati ES, Juca TL, et al. Proteome analysis of secondary somatic embryogenesis in cassava (Manihot esculenta). Plant Sci. 2008;175:717-23.

21. Zeng F, Zhang $X$, Cheng L, Hu L, Zhu L, Cao J, et al. A draft gene regulatory network for cellular totipotency reprogramming during plant somatic embryogenesis. Genomics. 2007;90:620-8.

22. Xiang D, Venglat $P$, Tibiche $C$, Yang H, Risseeuw E, Cao Y, et al. Genome-wide analysis reveals gene expression and metabolic network dynamics during embryo development in Arabidopsis. Plant Physiol. 2011;156:346-56.

23. $\mathrm{Xu} \mathrm{H}, \mathrm{Gao}$ Y, Wang J. Transcriptomic analysis of rice (Oryza sativa) developing embryos using the RNA-Seq technique. PLoS One. 2012;7:e30646.

24. Lu X, Chen D, Shu D, Zhang Z, Wang W, Klukas C, et al. The differentia transcription network between embryo and endosperm in the early developing maize seed. Plant Physiol. 2013;162:440-55.

25. Spencer MW, Casson SA, Lindsey K. Transcriptional profiling of the Arabidopsis embryo. Plant Physiol. 2007;143:924-40.

26. Belmonte MF, Kirkbride RC, Stone SL, Pelletier JM, Bui AQ, Yeung EC, et al. Comprehensive developmental profiles of gene activity in regions and subregions of the Arabidopsis seed. Proc Natl Acad Sci USA. 2013:110:E435-44.

27. Vandepoele K, Raes J, De Veylder L, Rouze P, Rombauts S, Inze D. Genome-wide analysis of core cell cycle genes in Arabidopsis. Plant Cell. 2002;14:903-16.

28. Menges M, de Jager SM, Gruissem W, Murray JAH. Global analysis of the core cell cycle regulators of Arabidopsis identifies novel genes, reveals multiple and highly specific profiles of expression and provides a coherent model for plant cell cycle control. Plant J. 2005;41:546-66.

29. Montero-Cortés M, Rodríguez-Paredes F, Burgeff C, Pérez-Nuñez T, Córdova I, Oropeza $C$, et al. Characterisation of a cyclin-dependent kinase (CDKA) gene expressed during somatic embryogenesis of coconut palm. Plant Cell Tiss Organ Cult. 2010;102:251-8.

30. Hemerly AS, Ferreira PC, Van Montagu M, Engler G, Inze D. Cell division events are essential for embryo patterning and morphogenesis: studies on dominant-negative cdc2aAt mutants of Arabidopsis. Plant J. 2000;23:123-30.

31. Iwakawa H, Shinmyo A, Sekine M. Arabidopsis CDKA;1, a cdc2 homologue, controls proliferation of generative cells in male gametogenesis. Plant J. 2006:45:819-31.

32. Feher A, Pasternak TP, Dudits D. Transition of somatic plant cells to an embryogenic state. Plant Cell Tiss Organ Cult. 2003;74:201-28.

33. Ronceret A, Guilleminot J, Lincker F, Gadea-Vacas J, Delorme V, Bechtold N, et al. Genetic analysis of two Arabidopsis DNA polymerase epsilon subunits during early embryogenesis. Plant J. 2005;44:223-36.

34. Liu Q, Gong ZZ. The coupling of epigenome replication with DNA replication. Curr Opin Plant Biol. 2011;14:187-94.

35. Masuda HP, Ramos GBA, de Almeida-Engler J, Cabral LM, Coqueiro VM, Macrini CMT, et al. Genome based identification and analysis of the prereplicative complex of Arabidopsis thaliana. FEBS Lett. 2004;574:192-202.

36. Krishnaswamy S, Verma S, Rahman MH, Kav NNV. Functional characterization of four APETALA2-family genes (RAP2.6, RAP2.6 L, DREB19 and DREB26) in Arabidopsis. Plant Mol Biol. 2011;75:107-27.

37. Rashotte AM, Mason MG, Hutchison CE, Ferreira FJ, Schaller GE, Kieber JJ. A subset of Arabidopsis AP2 transcription factors mediates cytokinin responses in concert with a two-component pathway. Proc Natl Acad Sci USA. 2006;103:11081-5.

38. Gliwicka M, Nowak K, Balazadeh S, Mueller-Roeber B, Gaj MD. Extensive modulation of the transcription factor transcriptome during somatic embryogenesis in Arabidopsis thaliana. PLoS One. 2013;8:e69261.

39. Stone SL, Kwong LW, Yee KM, Pelletier J, Lepiniec L, Fischer RL, et al. LEAFY COTYLEDON2 encodes a B3 domain transcription factor that induces embryo development. Proc Natl Acad Sci USA. 2001;98:11806-11.

40. Ikeda M, Umehara M, Kamada H. Embryogenesis related genes; its expression and roles during somatic and zygotic embryogenesis in carrot and Arabidopsis. Plant Biotech. 2006;23:153-61.

41. Boutilier K, Offringa R, Sharma VK, Kieft H, Ouellet T, Zhang L, et al. Ectopic expression of $\mathrm{BABY} B O O M$ triggers a conversion from vegetative to embryonic growth. Plant Cell. 2002;14:1737-49.

42. Heck GR, Perry SE, Nichols KW, Fernandez DE. AGL15, a MADS domain protein expressed in developing embryos. Plant Cell. 1995;7:1271-82. 
43. Hecht V, Vielle-Calzada JP, Hartog MV, Schmidt ED, Boutilier K, Grossniklaus $U$, et al. The Arabidopsis SOMATIC EMBRYOGENESIS RECEPTOR KINASE 1 gene is expressed in developing ovules and embryos and enhances embryogenic competence in culture. Plant Physiol. 2001;127:803-16.

44. Nuccio ML, Thomas TL. ATS1 and ATS3: two novel embryo-specific genes in Arabidopsis thaliana. Plant Mol Biol. 1999;39:1153-63.

45. Tammen SA, Friso S, Choi SW. Epigenetics: the link between nature and nurture. Mol Aspects Med. 2013;34:753-64

46. Furner IJ, Matzke M. Methylation and demethylation of the Arabidopsis genome. Curr Opin Plant Biol. 2010;14:137-41.

47. Xiao W, Custard KD, Brown RC, Lemmon BE, Harada JJ, Goldberg RB, et al. DNA methylation is critical for Arabidopsis embryogenesis and seed viability. Plant Cell. 2006:18:805-14.

48. Cao XF, Jacobsen SE. Role of the Arabidopsis DRM methyltransferases in de novo DNA methylation and gene silencing. Curr Biol. 2002;12:1138-44.

49. Huang JJ, Wang HH, Xie XJ, Zhang D, Liu Y, Guo GQ. Roles of DNA methyltransferases in Arabidopsis development. Afr J Biotechnol. 2010;9:8506-14.

50. Woo HR, Dittmer TA, Richards EJ. Three SRA-domain methylcytosine-binding proteins cooperate to maintain global CpG methylation and epigenetic silencing in Arabidopsis. PLoS Genet. 2008:4:e1000156.

51. Ortega-Galisteo AP, Morales-Ruiz T, Ariza RR, Roldan-Arjona T. Arabidopsis DEMETER-LIKE proteins DML2 and DML3 are required for appropriate distribution of DNA methylation marks. Plant Mol Biol. 2008:67:671-81.

52. Verbsky ML, Richards EJ. Chromatin remodeling in plants. Curr Opin Plant Biol. 2001;4:494-500.

53. Bordoli L, Netsch M, Luthi U, Lutz W, Eckner R. Plant orthologs of p300/CBP: conservation of a core domain in metazoan p300/CBP acetyltransferase-related proteins. Nucleic Acids Res. 2001;29:589-97.

54. Yao TP, Oh SP, Fuchs M, Zhou ND, Ch'ng LE, Newsome D, et al. Gene dosage-dependent embryonic development and proliferation defects in mice lacking the transcriptional integrator p300. Cell. 1998;93:361-72.

55. Earley KW, Shook MS, Brower-Toland B, Hicks L, Pikaard CS. In vitro specificities of Arabidopsis co-activator histone acetyltransferases: implications for histone hyperacetylation in gene activation. Plant J. 2007:52:615-26.

56. Deng W, Liu C, Pei Y, Deng X, Niu L, Cao X. Involvement of the histone acetyltransferase AtHAC1 in the regulation of flowering time via repression of FLOWERING LOCUS C in Arabidopsis. Plant Physiol. 2007;143:1660-8.

57. Pandey R, Muller A, Napoli CA, Selinger DA, Pikaard CS, Richards EJ, et al. Analysis of histone acetyltransferase and histone deacetylase families of Arabidopsis thaliana suggests functional diversification of chromatin modification among multicellular eukaryotes. Nucleic Acids Res. 2002;30:5036-55.

58. Hollender C, Liu ZC. Histone deacetylase genes in Arabidopsis development. Integr Plant Biol. 2008;50:875-85.

59. Zhou C, Labbe H, Sridha S, Wang L, Tian L, Latoszek-Green M, et al. Expression and function of HD2-type histone deacetylases in Arabidopsis development. Plant J. 2004;38:715-24.

60. Gaj MD. Direct somatic embryogenesis as a rapid and efficient system for in vitro regeneration of Arabidopsis thaliana. Plant Cell Tiss Organ Cult. 2001;64:39-46.

61. Trapnell C, Roberts A, Goff L, Pertea G, Kim D, Kelley DR, et al. Differential gene and transcript expression analysis of RNA-seq experiments with TopHat and Cufflinks. Nat Protoc. 2012;7:562-78.

62. Van Bel M, Proost S, Wischnitzki E, Movahedi S, Scheerlinck C, Van de Peer Y, et al. Dissecting plant genomes with the PLAZA comparative genomics platform. Plant Physiol. 2012;158:590-600.

63. Bindea G, Mlecnik B, Hackl H, Charoentong P, Tosolini M, Kirilovsky A, et al. ClueGO: a Cytoscape plug-in to decipher functionally grouped gene ontology and pathway annotation networks. Bioinformatics. 2009;25:1091-3.

\section{Submit your next manuscript to BioMed Central and take full advantage of:}

- Convenient online submission

- Thorough peer review

- No space constraints or color figure charges

- Immediate publication on acceptance

- Inclusion in PubMed, CAS, Scopus and Google Scholar

- Research which is freely available for redistribution

Submit your manuscript at www.biomedcentral.com/submit 